TRANSACTIONS OF THE

AMERICAN MATHEMATICAL SOCIETY

Volume 363, Number 7, July 2011, Pages 3573-3599

S 0002-9947(2011)05179-2

Article electronically published on January 28, 2011

\title{
CALABI-YAU THREE-FOLDS AND MODULI OF ABELIAN SURFACES II
}

\author{
MARK GROSS AND SORIN POPESCU
}

\begin{abstract}
We give explicit descriptions of the moduli spaces of abelian surfaces with polarizations of type $(1, d)$, for $d=12,14,16,18$ and 20. More precisely, in each case we show that a certain choice of moduli space of such abelian surfaces with a partial level structure can be described explicitly and is unirational, and in some cases rational. These moduli spaces with partial level structure are covers of the ordinary moduli spaces, so the Kodaira dimension of the ordinary moduli spaces in these cases is $-\infty$. In addition, we give a few new examples of Calabi-Yau three-folds fibred in abelian surfaces. In the case of $d=20$, such Calabi-Yau three-folds play a key role in the description of the abelian surfaces.
\end{abstract}

\section{INTRODUCTION}

The main goal of this paper, which is a continuation of [15, 16] and [17, is to describe birational models for moduli spaces $\mathcal{A}_{d}$ of polarized abelian surfaces of type $(1, d)$ for small values of $d$ and for moduli spaces of such polarized abelian surfaces with suitably defined partial or canonical level structure. We can then decide the uniruledness, unirationality or rationality of non-singular models of compactifications of these moduli spaces (which are quasi-projective three-folds, possibly singular). Since these properties are birational invariants, this determines the corresponding properties of those moduli spaces.

In the sequel we will use definitions and notation as in [15, [16, 17. See also 24, 22 and [19] for basic facts concerning abelian varieties and their moduli, as well as for the definition of a canonical level structure. We will also make use of partial level structures which are introduced and described in $\S 1$.

Using a version of the Maass-Kurokawa lifting, Gritsenko [12, 13] proved the existence of weight 3 cusp forms with respect to the paramodular group $\Gamma_{d}$, for almost all values of $d$. Since one knows the dimension for the space of Jacobi cusp forms, one deduces lower bounds for the dimensions of the spaces of cusp forms with respect to the paramodular group $\Gamma_{d}$. More precisely Gritsenko has shown that $\mathcal{A}_{d}$ is not unirational (in fact $p_{g}\left(\tilde{\mathcal{A}}_{d}\right) \geq 1$ ) if $d \geq 13$ and $d \neq 14,15,16,18,20,24,30$, 36 .

Moreover, a combination of the results in [12] and [20] shows that $\mathcal{A}_{p}^{\text {lev }}$ is of general type for all primes $p \geq 37$.

Received by the editors April 21, 2009 and, in revised form, August 4, 2009.

2000 Mathematics Subject Classification. Primary 14K10, 14J32.

This work was partially supported by NSF grants DMS-0805328, DMS-0502070, DMS-0083361, and MSRI, Berkeley. 
In this paper, we will focus on some of the moduli spaces excluded from Gritsenko's analysis. In particular, we define certain moduli spaces $\mathcal{A}_{d}^{H}$ of abelian surfaces with partial level structure, determined by groups $H$, which fit in between $\mathcal{A}_{d}^{\text {lev }}$ and $\mathcal{A}_{d}$ : there are forgetful maps

$$
\mathcal{A}_{d}^{\text {lev }} \rightarrow \mathcal{A}_{d}^{H} \rightarrow \mathcal{A}_{d}
$$

We will give details as to the structure of $\mathcal{A}_{d}$ (or rather $\mathcal{A}_{d}^{H}$ for certain choices of $H$ depending on the case) for $d=12,14,16,18$ and 20 . In particular, we will prove the following:

Theorem 0.1. For suitable choices of $H$, depending on the case,

(1) $\mathcal{A}_{12}^{\text {lev }}$ is birational to a rational singular complete intersection of two quadrics in $\mathbf{P}^{5}$.

(2) $\mathcal{A}_{14}^{H}$ is birational to the complete intersection of the standard Plücker quadric and the secant variety of the Veronese surface in $\mathbf{P}^{5}$. In particular it is unirational, being a conic bundle with a rational 2-section. It is not rational.

(3) $\mathcal{A}_{16}^{H}$ is birational to $\mathbf{P}^{1} \times \mathbf{P}^{1} \times \mathbf{P}^{1}$, and thus rational.

(4) $\mathcal{A}_{18}^{H}$ is birational to a rational singular complete intersection of 2 quadrics in $\mathbf{P}^{5}$.

(5) $\mathcal{A}_{20}^{H}$ is rational.

The basic method of proof for $d=12,14,16$ and 18 is as follows. For $d$ even, let $Z_{d}=\bigcup_{A} A \cap \mathbf{P}_{-}$, where the union runs over all Heisenberg invariant abelian surfaces of degree $(1, d)$ in $\mathbf{P}^{d-1}$ and $\mathbf{P}_{-}$is the -1 eigenspace of the standard involution $\iota: \mathbf{P}^{d-1} \rightarrow \mathbf{P}^{d-1}$ inducing negation on $A$. For each such $A, A \cap \mathbf{P}_{-}$ consists of the four odd two-torsion points of $A$, and these four points form an orbit under the $\mathbf{Z}_{2} \times \mathbf{Z}_{2}$ action on $\mathbf{P}_{-}$induced by $\sigma^{d / 2}$ and $\tau^{d / 2}$, where $\sigma$ and $\tau$ are the standard generators of the Heisenberg group $\mathbf{H}_{d}$. It was proved in [15] that each such orbit in $Z_{d}$ is, in general, the set of odd 2-torsion points of a unique Heisenberg invariant abelian surface $A$, so that $Z_{d} / \mathbf{Z}_{2} \times \mathbf{Z}_{2}$ is birational to $\mathcal{A}_{d}^{\text {lev }}$. In the $d=12$ case, this quotient can be described explicitly. In higher degrees, it is unlikely that this quotient is of negative Kodaira dimension, and in each of the higher degrees 14, 16 and 18, some additional particular geometry of the situation is used to take a further quotient of $Z_{d} / \mathbf{Z}_{2} \times \mathbf{Z}_{2}$ to obtain a variety birational to $\mathcal{A}_{d}^{H}$. The meaning of this moduli space is described in $\S 1$.

The case $d=20$ is treated differently. Here we use the fact that there exist singular quintics in $\mathbf{P}^{4}$ which contain pencils of (non-minimal) $(1,20)$-polarized abelian surfaces. This yields a ruling of $\mathcal{A}_{20}^{H}$.

Of course such quintics are Calabi-Yau three-folds, and there exist small resolutions of these quintics giving a non-singular Calabi-Yau with Hodge number $h^{1,1}=2$ and $h^{1,2}=2$. This adds to the stable of examples of Calabi-Yau threefolds containing a pencil of abelian surfaces found in [17. These examples have recently been of interest to physicists looking for interesting compactifications of space-time; see the work of Candelas and Davies [9] and Bak, Bouchard and Donagi [4.

In fact, this Calabi-Yau three-fold was the first of the examples discovered in this project, found by the second author in [27] while working on the classification of surfaces in $\mathbf{P}^{4}$. We realised that the existence of this Calabi-Yau proved uniruledness of $\mathcal{A}_{20}$, so we embarked on a search for other such Calabi-Yau three-folds, which led to the project of which this is the final part. 
There is one other example of a Calabi-Yau three-fold arising in this paper, a linear section of $\operatorname{Gr}(2,7)$, containing a pencil of $(1,14)$ abelian surfaces. In fact it turns out to be birational to the Pfaffian Calabi-Yau of degree 14 in $\mathbf{P}^{6}$ containing a pencil of $(1,7)$ abelian surfaces. There is some beautiful geometry associated to this Calabi-Yau which we discuss in $\S 3$.

\section{Preliminaries}

We review our notation and conventions concerning abelian surfaces; more details can be found in 15 .

Let $(A, \mathcal{L})$ be a general abelian surface with a polarization of type $(1, d)$. If $d \geq 5$, then $|\mathcal{L}|$ induces an embedding of $A \subset \mathbf{P}^{d-1}=\mathbf{P}\left(H^{0}(\mathcal{L})^{\vee}\right)$ of degree $2 d$. The line bundle $\mathcal{L}$ induces a natural map from $A$ to its dual $\hat{A}, \phi_{\mathcal{L}}: A \longrightarrow \hat{A}$, given by $x \mapsto t_{x}^{*} \mathcal{L} \otimes \mathcal{L}^{-1}$, where $t_{x}: A \longrightarrow A$ is the morphism given by translation by $x \in A$. Its kernel $K(\mathcal{L})$ is isomorphic to $\mathbf{Z}_{d} \times \mathbf{Z}_{d}$ and is dependent only on the polarization $c_{1}(\mathcal{L})$.

For every $x \in K(\mathcal{L})$ there is an isomorphism $t_{x}^{*} \mathcal{L} \cong \mathcal{L}$. This induces a projective representation $K(\mathcal{L}) \longrightarrow \operatorname{PGL}\left(H^{0}(\mathcal{L})\right)$, which lifts uniquely to a linear representation after taking a central extension of $K(\mathcal{L})$

$$
1 \longrightarrow \mathbf{C}^{*} \longrightarrow \mathcal{G}(\mathcal{L}) \longrightarrow K(\mathcal{L}) \longrightarrow 0
$$

whose Schur commutator map is the Weil pairing. $\mathcal{G}(\mathcal{L})$ is the theta group of $\mathcal{L}$ and is isomorphic to the abstract Heisenberg group $\mathcal{H}(1, d)$, while the above linear representation is isomorphic to the Schrödinger representation of $\mathcal{H}(1, d)$ on $V=\mathbf{C}\left(\mathbf{Z}_{d}\right)$, the vector space of complex-valued functions on $\mathbf{Z}_{d}$. An isomorphism between $\mathcal{G}(\mathcal{L})$ and $\mathcal{H}(1, d)$ which restricts to the identity on centers induces a symplectic isomorphism between $K(\mathcal{L})$ and $K(1, d)=\mathbf{Z}_{d} \times \mathbf{Z}_{d}$. This is an isomorphism which takes the Weil pairing on $K(\mathcal{L})$ to the standard skew-symmetric pairing $e^{(1, d)}$ on $K(1, d)$ with values in $\mathbf{C}^{*}$ given by

$$
e^{(1, d)}((1,0),(0,1))=\exp (-2 \pi i / d) .
$$

Such an isomorphism is called a level structure of canonical type on $\left(A, c_{1}(\mathcal{L})\right)$. (See [22], Chapter $8, \S 3$ or [15, $\S 1$.

A decomposition $K(\mathcal{L})=K_{1}(\mathcal{L}) \oplus K_{2}(\mathcal{L})$, with $K_{1}(\mathcal{L}) \cong K_{2}(\mathcal{L}) \cong \mathbf{Z}_{d}$ isotropic subgroups with respect to the Weil pairing, and a choice of a characteristic $c$ (22], Chapter $3, \S 1$ ) for $\mathcal{L}$, define a unique natural basis $\left\{\vartheta_{x}^{c} \mid x \in K_{1}(\mathcal{L})\right\}$ of canonical theta functions for the space $H^{0}(\mathcal{L})$ (see [25] and [22], Chapter 3, §2). This basis allows an identification of $H^{0}(\mathcal{L})$ with $V$ via $\vartheta_{\gamma}^{c} \mapsto x_{\gamma}$, where $x_{\gamma}$ is the function on $\mathbf{Z}_{d}$ defined by $x_{\gamma}(\delta)=\left\{\begin{array}{ll}1, & \gamma=\delta \\ 0, & \gamma \neq \delta\end{array}\right.$ for $\gamma, \delta \in \mathbf{Z}_{d}$. The functions $x_{0}, \ldots, x_{d-1}$ can also be identified with coordinates on $\mathbf{P}\left(H^{0}(\mathcal{L})^{\vee}\right)$. Under this identification, the representation $\mathcal{G}(\mathcal{L}) \longrightarrow \operatorname{GL}\left(H^{0}(\mathcal{L})\right)$ coincides with the Schrödinger representation $\mathcal{H}(1, d) \longrightarrow \mathrm{GL}(V)$. We will only consider the action of $\mathbf{H}_{d}$, the finite subgroup of $\mathcal{H}(1, d)$ generated in the Schrödinger representation by $\sigma$ and $\tau$, where

$$
\sigma\left(x_{i}\right)=x_{i-1}, \quad \tau\left(x_{i}\right)=\xi^{-i} x_{i},
$$

for all $i \in \mathbf{Z}_{d}$, and where $\xi=e^{\frac{2 \pi i}{d}}$ is a primitive root of unity of order $d$. Notice that $[\sigma, \tau]=\xi^{-1}$, so $\mathbf{H}_{d}$ is a central extension

$$
1 \longrightarrow \mu_{\mathbf{d}} \longrightarrow \mathbf{H}_{d} \longrightarrow \mathbf{Z}_{d} \times \mathbf{Z}_{d} \longrightarrow 0 \text {. }
$$


Therefore the choice of a canonical level structure means that if $A$ is embedded in $\mathbf{P}\left(H^{0}(\mathcal{L})^{\vee}\right)$ using as coordinates $x_{\gamma}=\vartheta_{\gamma}^{c}, \gamma \in \mathbf{Z}_{d}$, then the image of $A$ will be invariant under the action of the Heisenberg group $\mathbf{H}_{d}$ via the Schrödinger representation. (See [22], Chapter $6, \S 7$ for details.)

Moreover, if the line bundle $\mathcal{L}$ is chosen to be symmetric (and there are always finitely many choices of such an $\mathcal{L}$ for a given polarization type), then the embedding via $|\mathcal{L}|$ is also invariant under the involution $\iota$, where

$$
\iota\left(x_{i}\right)=x_{-i}, \quad i \in \mathbf{Z}_{d} .
$$

This involution restricts to $A$ as the involution $x \mapsto-x$. We will denote by $\mathbf{P}_{+}$and $\mathbf{P}_{-}$the $(+1)$ - and $(-1)$-eigenspaces of the involution $\iota$, respectively. We will also write as usual $\mathbf{H}_{d}^{e}:=\mathbf{H}_{d} \rtimes\langle\iota\rangle$.

We also recall a key result from [15]: In that paper, on $\mathbf{P}^{2 d-1} \times \mathbf{P}^{2 d-1}$, we have introduced a matrix

$$
M_{d}(x, y)=\left(x_{i+j} y_{i-j}+x_{i+j+d} y_{i-j+d}\right)_{0 \leq i, j \leq d-1}
$$

where the indices of the variables $x$ and $y$ above are all modulo $2 d$. This matrix has the property that if $A \subseteq \mathbf{P}^{2 d-1}$ is a Heisenberg invariant $(1,2 d)$-polarized abelian surface, then $M_{d}$ has rank at most two on $A \times A \subseteq \mathbf{P}^{2 d-1} \times \mathbf{P}^{2 d-1}$. Similarly, if $A \subseteq \mathbf{P}^{2 d}$ is a Heisenberg invariant $(1,2 d+1)$-polarized abelian surface, then the (Moore) matrix

$$
M_{2 d+1}^{\prime}(x, y)=\left(x_{d(i+j)} y_{d(i-j)}\right)_{i \in \mathbf{Z}_{2 d+1}, j \in \mathbf{Z}_{2 d+1}}
$$

on $\mathbf{P}^{2 d} \times \mathbf{P}^{2 d}$ has rank at most four on $A \times A \subseteq \mathbf{P}^{2 d} \times \mathbf{P}^{2 d}$. These matrices will prove to be ubiquitous!

Partial level structures. Recall from above that a canonical level structure on a polarized abelian surface $(A, \mathcal{L})$ with polarization of type $D=\left(d_{1}, d_{2}\right)$ is a symplectic isomorphism $b: K(\mathcal{L}) \rightarrow K(D)$. (See also [15], $\S 1.3$ and [17] for notation and details.)

Definition 1.1. Let $H \subseteq K(D)$ be a fixed subgroup. An $H$-level structure on a $D$-polarized abelian surface $(A, \mathcal{L})$ is an $H$-equivalence class of canonical level structures [b], where two canonical level structures $b_{1}$ and $b_{2}$ are $H$-equivalent if there is a symplectic automorphism $\phi: K(D) \rightarrow K(D)$ with $\left.\phi\right|_{H}=i d_{H}$ and $\phi \circ b_{1}=b_{2}$.

The following proposition uses the notation of [15], $\S 1.3$.

Proposition 1.2. Let $X_{Z}$ and $X_{Z^{\prime}}$ be two fibres of $\mathcal{X}_{D} \rightarrow \mathcal{H}_{g}$, and suppose $\left.\mathcal{L}\right|_{X_{Z}}$ and $\left.\mathcal{L}\right|_{X_{Z}^{\prime}}$ are both very ample, so we can identify $X_{Z}$ and $X_{Z^{\prime}}$ with their images in $\mathbf{P}(V)$, where $V=H^{0}\left(\left.\mathcal{L}\right|_{X_{Z}}\right)=H^{0}\left(\left.\mathcal{L}\right|_{X_{Z^{\prime}}}\right)$. Let $H \subseteq K(D)$ be a subgroup, and let $H^{\prime}$ be the inverse image of $H$ in $\mathcal{H}(D)$. We will think of $H^{\prime}$ as $H^{\prime} \subseteq \mathrm{GL}(V)$ via the Schrödinger representation of $\mathcal{H}(D)$. Then the canonical level structures on $X_{Z}$ and $X_{Z^{\prime}}$ are $H$-equivalent if and only if there exists an element $T \in N(\mathcal{H}(D))$, the normalizer of $\mathcal{H}(D)$ in $\mathrm{GL}(V)$, such that $[T, \alpha]=T \alpha T^{-1} \alpha^{-1} \in \mathbf{C}^{*}$ for all $\alpha \in H^{\prime}$, and $T\left(X_{Z}\right)=X_{Z^{\prime}}$.

Proof. Suppose that the level structures $b, b^{\prime}$ on $X_{Z}$ and $X_{Z^{\prime}}$ are $H$-equivalent. Then there exists a symplectic automorphism $\phi \in S p(D)$ with $\phi \circ b=b^{\prime}$ and $\left.\phi\right|_{H}=i d$. We have an exact sequence

$$
0 \rightarrow \mathcal{H}(D) \rightarrow N(\mathcal{H}(D)) \rightarrow S p(D) \rightarrow 1
$$


where $N(\mathcal{H}(D))$ is the normalizer of $\mathcal{H}(D)$ in $\mathrm{GL}(V)$. Lift $\phi$ to an element $T \in$ $N(\mathcal{H}(D))$; then $T\left(X_{Z}\right)=X_{Z^{\prime}}$, by [15], Proposition 1.3.1. Also, the action of $\phi$ on $K(D)$ is given by conjugation by $T$, so for any $\alpha \in H^{\prime}, T \alpha T^{-1}$ must be a multiple of $\alpha$, so $T \alpha T^{-1} \alpha^{-1} \in \mathbf{C}^{*}$ for all $\alpha \in H^{\prime}$.

Conversely, if there exists a transformation $T \in N(\mathcal{H}(D))$ with $T \alpha T^{-1} \alpha^{-1} \in \mathbf{C}^{*}$ for all $\alpha \in H^{\prime}$, and $T\left(X_{Z}\right)=X_{Z^{\prime}}$, then $X_{Z}$ and $X_{Z^{\prime}}$ are isomorphic as polarized abelian surfaces. $T$ then induces an element of $S p(D)$ which is the identity on $H$, so that the canonical level structures on $X_{Z}$ and $X_{Z^{\prime}}$ are $H$-equivalent.

With notation as in Proposition 1.2 we now introduce the following:

Definition 1.3. If $H \subseteq K(D)$ is a subgroup and $\iota: V \rightarrow V$ the usual involution, define $\mathrm{GL}_{H} \subseteq \mathrm{GL}(V)$ by

$$
\mathrm{GL}_{H}=\left\{T \in \mathrm{GL}(V) \mid T \alpha T^{-1} \alpha^{-1} \in \mathbf{C}^{*} \text { for all } \alpha \in H^{\prime} \text { and } \iota T=T \iota\right\},
$$

where $H^{\prime}$ is as in Proposition 1.2

In two special cases relevant for us we can explicitly describe $\mathrm{GL}_{H}$ :

Proposition 1.4. Suppose $D=(1,2 d)$.

(1) If $H \subseteq K(D)$ is the subgroup generated by $\sigma^{2}$ and $\tau$, then $T \in \mathrm{GL}_{H}$ if and only if either $T=\operatorname{diag}(a, b, a, b, \ldots, a, b)$ or $T=\sigma^{d} \circ \operatorname{diag}(a, b, a, b, \ldots, a, b)$ for some $a, b \in \mathbf{C}^{*}$.

(2) If $H=2 K(D)$, then $T=\left(a_{i j}\right) \in \mathrm{GL}(V)$ is in $\mathrm{GL}_{H}$ if and only if $a_{i j}=0$ unless $i=j$ or $i=j+d$, and $a_{i+2, j+2}=a_{i, j}$.

(3) In either of the above two cases, if $T \in \mathrm{GL}(V)$ satisfies $T \alpha T^{-1} \alpha^{-1} \in \mathbf{C}^{*}$ for all $\alpha \in H^{\prime}$, then there exists a,b such that, with $T^{\prime}=\sigma^{a} \tau^{b} T$, we have $T^{\prime} \in \mathrm{GL}_{H}$.

Proof. Note that if $T=\left(a_{i j}\right)$, and $T \circ \sigma^{2}=C_{1} \sigma^{2} \circ T$ for some $C_{1} \in \mathbf{C}^{*}$, then

$$
a_{i-2, j-2}=C_{1} a_{i j} \quad \forall i, j \in \mathbf{Z} / 2 d \mathbf{Z} \text {. }
$$

Note that assuming $a_{i j} \neq 0$ for some $i, j$, using this $d$ times implies $C_{1}^{d}=1$, i.e., $C_{1}=\xi^{2 n}$ for some $n \in \mathbf{Z} / 2 d \mathbf{Z}$, where $\xi$ is a fixed primitive $2 d$ th root of unity. If $T \circ \tau=C_{2} \tau \circ T$, then

$$
\xi^{-j} a_{i j}=C_{2} \xi^{-i} a_{i j} \quad \forall i, j \in \mathbf{Z} / 2 d \mathbf{Z},
$$

while if $T \circ \tau^{2}=C_{3} \tau^{2} \circ T$, then

$$
\xi^{-2 j} a_{i j}=C_{3} \xi^{-2 i} a_{i j} \quad \forall i, j \in \mathbf{Z} / 2 d \mathbf{Z} .
$$

Finally, if $T \circ \iota=\iota \circ T$, then

$$
a_{i j}=a_{2 d-i, 2 d-j} .
$$

If (1.1) and (1.2) hold for $T$, then we see from (1.2) that either $a_{i j}=0$ or $C_{2}=\xi^{i-j}$. Thus $C_{2}$ must be of the form $C_{2}=\xi^{m}$ for some $m \in \mathbf{Z} / 2 d \mathbf{Z}$, and then $a_{i j}=0$ unless $i-j=m$.

Furthermore, if $T \in \mathrm{GL}_{H}$ for $H$ generated by $\sigma^{2}$ and $\tau$, then (1.4) implies $2 m \equiv 0 \bmod 2 d$, so $m=0$ or $d$, in which case $C_{2}=1$ or -1 . If $m=0$, then (1.1) and (1.4) applied to $(i, j)=(d+1, d+1)$ imply together that $C_{1}=1$ and $T=\operatorname{diag}(a, b, a, b, \ldots)$. If $m=d$, then (1.1) and (1.4) applied to $(i, j)=(1, d+1)$ imply together that $C_{1}=1$ and $T=\sigma^{d} \circ \operatorname{diag}(a, b, a, b, \ldots)$. This proves (1). 
For (3) in the case that $H$ is generated by $\sigma^{2}$ and $\tau$, note, from the properties of $T$ derived from (1.1) and (1.2), that if we set $T^{\prime}=\sigma^{m} \tau^{-n} T$ and if $\left\{x_{i} \mid i \in \mathbf{Z} / 2 d \mathbf{Z}\right\}$ is the standard basis of $V$ as usual, then

$$
\begin{aligned}
T^{\prime}\left(x_{i}\right) & =\sigma^{m} \tau^{-n}\left(a_{i+m, i} x_{i+m}\right) \\
& =\xi^{n(i+m)} a_{i+m, i} \sigma^{m}\left(x_{i+m}\right) \\
& =\xi^{n(i+m)} a_{i+m, i} x_{i}
\end{aligned}
$$

so the matrix $\left(a_{i j}^{\prime}\right)$ for $T^{\prime}$ is diagonal, and using (1.1) and $C_{1}=\xi^{2 n}$,

$$
\begin{aligned}
a_{i i}^{\prime}=\xi^{n(i+m)} a_{i+m, i}=\xi^{n(i+m)} \xi^{-2 n} a_{i+m-2, i-2} & =\xi^{n(i+m)} \xi^{-2 n} \xi^{-n(i+m-2)} a_{i-2, i-2}^{\prime} \\
& =a_{i-2, i-2}^{\prime} .
\end{aligned}
$$

Thus $T^{\prime} \in \mathrm{GL}_{H}$ by (1).

For (2), note that from (1.3), either $a_{i j}=0$ or $C_{3}=\xi^{2 i-2 j}$. Thus $C_{3}$ must be of the form $C_{3}=\xi^{2 m}$, and then $a_{i j}=0$ unless $2(i-j) \equiv 2 m \bmod 2 d$. But if $T \in \mathrm{GL}_{H}$, then (1.4) implies $4 m \equiv 0 \bmod 2 d$. We thus need to consider two cases:

$d$ odd: $2 m=0$, and $a_{i j}=0$ unless $i-j=0$ or $d$. Applying (1.4) to $(i, j)=$ $(d+1, d+1)$ and $(1, d+1)$ shows that $C_{1}=1$, and we have the desired form for $T$.

$d$ even: We have two cases: $2 m=0$ or $2 m=d$. In the first case, it follows as in the odd case that $T$ is of the desired form, and we need to rule out $2 m=d$. In this case, $a_{i j}=0$ unless $i-j=d / 2$ or $3 d / 2$. But note by (1.4) that $a_{0, d / 2}=a_{0,3 d / 2}$ and $a_{d, d / 2}=a_{d, 3 d / 2}$. These are the only possible non-zero entries in columns $d / 2$ and $3 d / 2$, and since these columns are then identical, $T$ cannot be invertible. Thus this case doesn't arise.

Finally, we show (3) in the case where $H$ is generated by $\sigma^{2}$ and $\tau^{2}$, so we assume $T$ is given satisfying (1.1) and (1.3). Again setting $T^{\prime}=\sigma^{m} \tau^{-n} T$, we see that

$$
\begin{aligned}
T^{\prime}\left(x_{i}\right) & =\sigma^{m} \tau^{-n}\left(a_{i+m, i} x_{i+m}+a_{i+m+d, i} x_{i+m+d}\right) \\
& =\xi^{n(i+m)} a_{i+m, i} x_{i}+\xi^{n(i+m+d)} a_{i+m+d, i} x_{i+d} .
\end{aligned}
$$

Thus if $T^{\prime}=\left(a_{i j}^{\prime}\right)$, then $a_{i j}^{\prime}=0$ unless $i=j$ or $i=j+d$. Furthermore, using (1.1) and $C_{1}=\xi^{2 n}$, with $\delta=0$ or $d$,

$$
\begin{aligned}
a_{i+\delta, i}^{\prime} & =\xi^{n(i+m+\delta)} a_{i+m+\delta, i}=\xi^{n(i+m+\delta)} \xi^{-2 n} a_{i+m+\delta-2, i-2} \\
& =\xi^{n(i+m+\delta)} \xi^{-2 n} \xi^{-n(i+m+\delta-2)} a_{i+\delta-2, i-2}^{\prime}=a_{i+\delta-2, i-2}^{\prime} .
\end{aligned}
$$

This shows from (2) that $T^{\prime} \in \mathrm{GL}_{H}$.

Continuing to focus on the $(1,2 d)$ case, recall from [15], $\S 6$, the set $Z_{2 d} \subseteq \mathbf{P}_{-}$, which is the union of odd 2-torsion points of Heisenberg invariant abelian surfaces of type $D=(1,2 d) . \overline{Z_{2 d}}$ is an irreducible three-fold for $d \geq 5$, as was proven in [15], $\S 6$.

Definition 1.5. $\mathcal{A}_{2 d}^{H}$ denotes the moduli space of $(1,2 d)$-polarized abelian surfaces with an $H$-level structure, for $H \subseteq K(D)$ a subgroup. In particular, if $H=$ $K(D)$, then $\mathcal{A}_{2 d}^{H}=\mathcal{A}_{2 d}^{\text {lev }}$, the moduli space of $(1,2 d)$-polarized abelian surfaces with canonical level structure.

The reason for introducing $\mathrm{GL}_{H}$ is the following theorem:

Theorem 1.6. If $d \geq 5$ and $H \subseteq K(D)$ is either generated by $\sigma^{2}$ and $\tau$ or is $2 K(D)$, then $\mathcal{A}_{2 d}^{H}$ is birationally equivalent to $\overline{Z_{2 d}} /\left(\mathrm{GL}_{H} \cap N(\mathcal{H}(1,2 d))\right)$. 
Proof. We know by [15], Theorem 6.2 that there is a birational map

$$
\Theta_{2 d}: \mathcal{A}_{2 d}^{\text {lev }}-\overline{Z_{2 d}} / \mathbf{Z}_{2} \times \mathbf{Z}_{2}
$$

taking $[A] \in \mathcal{A}_{2 d}^{\text {lev }}$ to the $\mathbf{Z}_{2} \times \mathbf{Z}_{2}=\left\langle\sigma^{d}, \tau^{d}\right\rangle$-orbit $A \cap \mathbf{P}_{-}$, the odd two-torsion points of $A$. One notes from Proposition 1.4 that $\sigma^{d}, \tau^{d} \in G L_{H} \cap N(\mathcal{H}(1,2 d))$, so that we get a factorization of the quotient

$$
\overline{Z_{2 d}} \rightarrow \overline{Z_{2 d}} / \mathbf{Z}_{2} \times \mathbf{Z}_{2} \rightarrow \overline{Z_{2 d}} /\left(G L_{H} \cap N(\mathcal{H}(1,2 d))\right) .
$$

In particular, the action of $G L_{H} \cap N(\mathcal{H}(1,2 d))$ descends to $\overline{Z_{2 d}} / \mathbf{Z}_{2} \times \mathbf{Z}_{2}$.

Letting $X_{Z}, X_{Z^{\prime}}$ be two general fibres of the universal family $\mathcal{X}_{(1,2 d)} \rightarrow \mathcal{H}_{2}$ as in Proposition 1.2. these represent the same point in $\mathcal{A}_{2 d}^{H}$ if and only if $T\left(X_{Z}\right)=X_{Z^{\prime}}$ for some $T \in N(\mathcal{H}(1,2 d))$ such that $T \alpha T^{-1} \alpha^{-1} \in \mathbf{C}^{*}$ for all $\alpha \in H^{\prime}$. Thus, if there exists $T \in G L_{H} \cap N(\mathcal{H}(1,2 d))$ such that $T\left(\Theta_{2 d}\left(\left[X_{Z}\right]\right)\right)=\Theta_{2 d}\left(\left[X_{Z^{\prime}}\right]\right)$, it follows that $T\left(X_{Z} \cap \mathbf{P}_{-}\right)=X_{Z^{\prime}} \cap \mathbf{P}_{-}$. Indeed, $T$ commutes with $\iota$, and hence takes odd two-torsion points of $X_{Z}$ to odd two-torsion points of $X_{Z^{\prime}}$. Since $T$ is in the normalizer of $\mathcal{H}(1,2 d), T\left(X_{Z}\right)$ is Heisenberg invariant, and by [15], Theorem 6.2, the general Heisenberg invariant abelian surface is determined by its intersection with $\mathbf{P}_{-}$. Thus $T\left(X_{Z}\right)=X_{Z^{\prime}}$ and $X_{Z}$ is $H$-equivalent to $X_{Z^{\prime}}$.

Conversely, if $X_{Z}$ is $H$-equivalent to $X_{Z^{\prime}}$, we obtain $T \in N(\mathcal{H}(1,2 d))$ with $T \alpha T^{-1} \alpha^{-1} \in \mathbf{C}^{*}$ for all $\alpha \in H^{\prime}$, but we need not have $T \iota=\iota T$. By Proposition 1.4. we have $T^{\prime}=\sigma^{a} \tau^{b} T$ which does satisfy $T^{\prime} \iota=\iota T^{\prime}$. So $T^{\prime} \in G L_{H} \cap N(\mathcal{H}(1,2 d))$ and $T^{\prime}\left(\left[X_{Z}\right]\right)=\left[X_{Z^{\prime}}\right]$. Thus $\overline{Z_{2 d}} /\left(G L_{H} \cap N(\mathcal{H}(1,2 d))\right)$ is birationally equivalent to $\mathcal{A}_{2 d}^{H}$, as desired.

\section{Moduli of $(1,12)$-Polarized ABelian surfaces}

Given the results of [15], $\S 2$ and $\S 6$, it is not difficult to describe the structure of $\mathcal{A}_{12}^{\text {lev }}$. By [15, Corollary 2.7, if $A \subseteq \mathbf{P}^{11}$ is an $\mathbf{H}_{12}$-invariant abelian surface with polarization of type $(1,12)$, with $y \in A$ a point, then $M_{6}(x, y)$ has rank at most two on $A$. So in particular, if $x \in A \cap \mathbf{P}_{-}^{4}$, the matrix $M_{6}(x, x)$ must have rank at most two. Now if $x \in \mathbf{P}_{-}^{4}, M_{6}(x, x)$ is a $6 \times 6$ skew-symmetric matrix, and thus $x \in A \cap \mathbf{P}_{-}^{4}$ for some Heisenberg invariant abelian surface implies that the $4 \times 4$ Pfaffians of $M_{6}(x, x)$ vanish.

For $x \in \mathbf{P}_{-}^{4}$ with coordinates

$$
\begin{aligned}
& \left(x_{1}: x_{2}: x_{3}: x_{4}: x_{5}\right) \\
& \quad\left(=\left(0: x_{1}: x_{2}: x_{3}: x_{4}: x_{5}: 0:-x_{5}:-x_{4}:-x_{3}:-x_{2}:-x_{1}\right) \in \mathbf{P}^{11}\right),
\end{aligned}
$$

the first $4 \times 4$ block of $M_{6}(x, x)$ is

$$
\left(\begin{array}{cccc}
0 & -x_{1}^{2}-x_{5}^{2} & -x_{2}^{2}-x_{4}^{2} & -2 x_{3}^{2} \\
x_{1}^{2}+x_{5}^{2} & 0 & -x_{1} x_{3}-x_{3} x_{5} & -2 x_{2} x_{4} \\
x_{2}^{2}+x_{4}^{2} & x_{1} x_{3}+x_{3} x_{5} & 0 & -2 x_{1} x_{5} \\
2 x_{3}^{2} & 2 x_{2} x_{4} & 2 x_{1} x_{5} & 0
\end{array}\right)
$$

and the $4 \times 4$ Pfaffian of this is

$$
f=2 x_{3}^{2}\left(x_{1} x_{3}+x_{3} x_{5}\right)-2\left(x_{2}^{2}+x_{4}^{2}\right) x_{2} x_{4}+2 x_{1} x_{5}\left(x_{1}^{2}+x_{5}^{2}\right) .
$$

Let $Q$ be the quartic hypersurface in $\mathbf{P}_{-}^{4}$ determined by the equation $f=0$.

Theorem 2.1. The map $\Theta_{12}: \mathcal{A}_{12}^{\text {lev }}-\rightarrow \mathbf{P}_{-}^{4} / \mathbf{Z}_{2} \times \mathbf{Z}_{2}$ induces a birational map to $Q / \mathbf{Z}_{2} \times \mathbf{Z}_{2}$. 
Proof. By [15], Theorem 6.2, $\Theta_{12}$ is birational onto its image. Let $\pi: \mathbf{P}_{-}^{4} \rightarrow$ $\mathbf{P}_{-}^{4} / \mathbf{Z}_{2} \times \mathbf{Z}_{2}$ be the projection, and let $Z_{12}$ be the inverse image under $\pi$ of im $\Theta_{12}$. Then by construction, $\overline{Z_{12}} \subseteq Q$. On the other hand, $\overline{Z_{12}}$ must be three-dimensional. It is easy to check that $Q$ is a non-singular quartic hypersurface, and thus $\overline{Z_{12}}=$ $Q$.

We now give a more precise description of $Q / \mathbf{Z}_{2} \times \mathbf{Z}_{2}$.

Theorem 2.2. $Q / \mathbf{Z}_{2} \times \mathbf{Z}_{2}$ is isomorphic to the complete intersection in $\mathbf{P}^{5}$ given by the equations

$$
\begin{aligned}
z_{0} z_{5}-z_{1} z_{4}+z_{2} z_{3} & =0, \quad \text { (the Plücker quadric) } \\
z_{0} z_{2}-z_{3}^{2}+2 z_{2} z_{5} & =0 .
\end{aligned}
$$

In particular, $Q / \mathbf{Z}_{2} \times \mathbf{Z}_{2}$ is rational.

Proof. We first note that the $\mathbf{Z}_{2} \times \mathbf{Z}_{2}$ action on $\mathbf{P}_{-}^{4}$ takes the form

$$
\begin{aligned}
& \sigma^{6}:\left(x_{1}: x_{2}: x_{3}: x_{4}: x_{5}\right) \mapsto\left(x_{5}: x_{4}: x_{3}: x_{2}: x_{1}\right), \\
& \tau^{6}:\left(x_{1}: x_{2}: x_{3}: x_{4}: x_{5}\right) \mapsto\left(x_{1}:-x_{2}: x_{3}:-x_{4}: x_{5}\right)
\end{aligned}
$$

and the ring of invariants of $\mathbf{C}\left[x_{1}, \ldots, x_{5}\right]$ under this action is generated by $x_{3}, x_{1}+$ $x_{5}, x_{1}^{2}+x_{5}^{2}, x_{2}^{2}+x_{4}^{2}$, and $x_{2} x_{4}$, and thus the invariant quadrics

$$
\begin{aligned}
z_{0}=x_{1}^{2}+x_{5}^{2}, & z_{3}=x_{1} x_{3}+x_{3} x_{5}, \\
z_{1}=x_{2}^{2}+x_{4}^{2}, & z_{4}=x_{2} x_{4}, \\
z_{2}=x_{3}^{2}, & z_{5}=x_{1} x_{5}
\end{aligned}
$$

generate the invariants of even degree of this action. Thus $\mathbf{P}_{-}^{4} / \mathbf{Z}_{2} \times \mathbf{Z}_{2}$ is isomorphic to a hypersurface in $\mathbf{P}^{5}=\operatorname{Proj} \mathbf{C}\left[z_{0}, \ldots, z_{5}\right]$. It is easy to see that the quadrics $z_{0}, \ldots, z_{5}$ satisfy the relation

$$
z_{0} z_{2}-z_{3}^{2}+2 z_{2} z_{5}=0 .
$$

On the other hand, $Q$ is given by

$$
f=2\left(z_{0} z_{5}-z_{1} z_{4}+z_{2} z_{3}\right) .
$$

This gives the desired equations.

Rationality of this three-fold can easily be seen by adapting the standard proof of rationality (see e.g. 11], pg. 796) of the non-singular quadric line complex to this singular one. One projects from a line contained in $Q / \mathbf{Z}_{2} \times \mathbf{Z}_{2}$ to $\mathbf{P}^{3}$. For example, one can use the line given by $z_{0}=z_{1}=z_{3}=z_{5}=0$.

\section{Moduli of $(1,14)$-POlarized AbELian SURFACES}

This is the first case considered here in which the locus of odd 2-torsion points (in $\left.\mathbf{P}_{-}\right)$is no longer of negative Kodaira dimension. Following the strategy for $(1,12)$, one finds that the matrix $M_{7}(x, x)$ has rank 2 on a quartic hypersurface $f_{1}=0$ in $\mathbf{P}_{-}^{5}$, and the matrix $M_{7}(\sigma(x), x)$ has rank 2 on another quartic hypersurface $f_{2}=0$. One finds $f_{1}=f_{2}=0$ is an irreducible three-fold, and hence is the closure of the locus of odd 2-torsion points, $\overline{Z_{14}}$ in the notation of [15], $\S 6$. This quotient is very likely of general type, but this is difficult to determine. So at this point, we need to stop considering the moduli space with full level structure. Instead, we will 
consider the subgroup $H \subseteq K(1,14)$ generated by $\sigma^{2}$ and $\tau$, and find the moduli space $\mathcal{A}_{14}^{H}$ of $(1,14)$ abelian surfaces with an $H$-level structure.

We will first study the geometry of certain mappings which will enable us to divide out $\overline{Z_{14}}$ by $\mathbf{Z}_{2} \times \mathbf{Z}_{2}$ as well as make the additional identifications which identify $H$-equivalent level structures. It will turn out that this is easier than computing $\overline{Z_{14}} / \mathbf{Z}_{2} \times \mathbf{Z}_{2}$.

More precisely, looking at the matrix $M_{7}(x, x)$ on $\mathbf{P}_{-}^{5}$, one sees that it contains a submatrix

$$
M^{\prime}=\left(\begin{array}{cccc}
0 & -x_{1}^{2}-x_{6}^{2} & -x_{2}^{2}-x_{5}^{2} & -x_{3}^{2}-x_{4}^{2} \\
x_{1}^{2}+x_{6}^{2} & 0 & -x_{1} x_{3}-x_{4} x_{6} & -x_{2} x_{4}-x_{3} x_{5} \\
x_{2}^{2}+x_{5}^{2} & x_{1} x_{3}+x_{4} x_{6} & 0 & -x_{1} x_{5}-x_{2} x_{6} \\
x_{3}^{2}+x_{4}^{2} & x_{2} x_{4}+x_{3} x_{5} & x_{1} x_{5}+x_{2} x_{6} & 0
\end{array}\right)
$$

which is rank $\leq 2$ on a quartic $Q_{1}=\left\{f_{1}=0\right\}$ where $f_{1}$ is the $4 \times 4$ Pfaffian of $M^{\prime}$. Similarly, $M_{7}(\sigma(x), x)$ restricted to $\mathbf{P}_{-}^{5}$ has a submatrix

$$
M_{\sigma}^{\prime}=\left(\begin{array}{cccc}
0 & -x_{1} x_{2}-x_{5} x_{6} & -x_{2} x_{3}-x_{4} x_{5} & -2 x_{3} x_{4} \\
x_{1} x_{2}+x_{5} x_{6} & 0 & -x_{1} x_{4}-x_{3} x_{6} & -2 x_{2} x_{5} \\
x_{2} x_{3}+x_{4} x_{5} & x_{1} x_{4}+x_{3} x_{6} & 0 & -2 x_{1} x_{6} \\
2 x_{3} x_{4} & 2 x_{2} x_{5} & 2 x_{1} x_{6} & 0
\end{array}\right) .
$$

$M_{\sigma}^{\prime}$ has rank 2 on the set $Q_{2}=\left\{f_{2}=0\right\}$, where $f_{2}$ is (one-half of) the Pfaffian of $M_{\sigma}^{\prime}$. Since $M_{\sigma}^{\prime}$ has rank 2 at the general point of $Q_{2}$, we obtain a rational map $\phi: Q_{2}-\rightarrow \operatorname{Gr}(2,4)$ induced by $M_{\sigma}^{\prime}$, taking a point of $Q_{2}$ to the kernel of $M_{\sigma}^{\prime}$ at that point. This is defined where $M_{\sigma}^{\prime}$ is non-zero, and in Plücker coordinates is given by

$$
\begin{aligned}
\left(x_{1}, \ldots, x_{6}\right) & \mapsto\left(z_{0}, \ldots, z_{5}\right) \\
\quad & =\left(x_{1} x_{2}+x_{5} x_{6}, x_{2} x_{3}+x_{4} x_{5}, x_{1} x_{4}+x_{3} x_{6}, 2 x_{3} x_{4}, 2 x_{2} x_{5}, 2 x_{1} x_{6}\right) .
\end{aligned}
$$

Here,

$$
f_{2}=x_{1} x_{3} x_{4}^{2}-x_{2}^{2} x_{3} x_{5}-x_{2} x_{4} x_{5}^{2}+x_{1}^{2} x_{2} x_{6}+x_{3}^{2} x_{4} x_{6}+x_{1} x_{5} x_{6}^{2}
$$

(this is in fact the unique bi-degree $(2,2)$ bi-invariant of $\operatorname{PSL}_{2}\left(\mathbf{Z}_{7}\right)$ acting on the variables $x_{1}, x_{3}, x_{5}$ and $x_{2}, x_{4}, x_{6}$ diagonally in its three-dimensional representation) and

$$
f_{1}-f_{2}=x_{1} x_{3}^{3}-x_{2}^{3} x_{4}+x_{1}^{3} x_{5}-x_{3} x_{5}^{3}+x_{4}^{3} x_{6}+x_{2} x_{6}^{3}
$$

(which is in fact the sum of two Klein quartics, one in the variables $x_{1}, x_{3}, x_{5}$ and the other in the variables $x_{2}, x_{4}, x_{6}$, the Klein quartic being the unique quartic invariant of $\mathrm{PSL}_{2}\left(\mathbf{Z}_{7}\right)$ in its three-dimensional representation).

Lemma 3.1. (1) $\phi$ is not defined on the union of the planes

$$
P_{1}=\left\{x_{1}=x_{3}=x_{5}=0\right\}
$$

and

$$
P_{2}=\left\{x_{2}=x_{4}=x_{6}=0\right\} .
$$

(2) The closure of the image of $\phi, \overline{\operatorname{im} \phi}$, is a complete intersection of type $(2,3)$ whose equations are the Plücker quadric $z_{0} z_{5}-z_{1} z_{4}+z_{2} z_{3}=0$ and the cubic

$$
\operatorname{det}\left(\begin{array}{ccc}
z_{5} & z_{2} & z_{0} \\
z_{2} & z_{3} & z_{1} \\
z_{0} & z_{1} & z_{4}
\end{array}\right)=0
$$


This latter hypersurface is the secant variety of the Veronese surface determined by the $2 \times 2$ minors of this matrix.

(3) $\mathrm{GL}_{H}$ acts on the fibres of $\phi$, and the fibre of $\phi$ over a general point is the closure of a $\mathrm{GL}_{H}$-orbit.

(4) $Q_{2}$ is irreducible.

(5) $\overline{\operatorname{im} \phi}$ is an irreducible, unirational 3 -fold.

Proof. (1) One easily checks that the zero locus of the six quadrics defining the map $\phi$ is $P_{1} \cup P_{2}$.

(2) First consider the rational map $\phi^{\prime}: \mathbf{P}_{-}^{5}-\rightarrow \mathbf{P}^{8}$ induced by the linear system $\left|H^{0}\left(\mathcal{I}_{P_{1} \cup P_{2}}(2)\right)\right|$. Specifically, using coordinates $\left\{z_{i j}\right\}$ on $\mathbf{P}^{8}$ with $i \in\{1,3,5\}$ and $j \in\{2,4,6\}$, we give $\phi^{\prime}$ via $z_{i j}=x_{i} x_{j}$. The equations of $\overline{\operatorname{im} \phi^{\prime}}$ are then given as the $2 \times 2$ minors of the matrix

$$
L=\left(\begin{array}{lll}
z_{16} & z_{14} & z_{12} \\
z_{36} & z_{34} & z_{32} \\
z_{56} & z_{54} & z_{52}
\end{array}\right)
$$

If $X$ is the blow-up of $\mathbf{P}_{-}^{5}$ along $P_{1} \cup P_{2}$, then $\phi^{\prime}$ lifts to a morphism $\tilde{\phi}^{\prime}: X \rightarrow \mathbf{P}^{8}$ which is easily seen to describe $X$ as a $\mathbf{P}^{1}$-bundle over $\overline{\operatorname{im} \phi^{\prime}}$, which is naturally isomorphic to $P_{1} \times P_{2}$ embedded via the Segre embedding. Explicitly, a line $l$ in $\mathbf{P}_{-}^{5}$ meeting $P_{1}$ and $P_{2}$ gets mapped to the point $\left(p_{1}, p_{2}\right)$ of $P_{1} \times P_{2}$ with $l \cap P_{1}=\left\{p_{1}\right\}$ and $l \cap P_{2}=\left\{p_{2}\right\}$.

Now $\phi$ is the composition of $\left.\phi^{\prime}\right|_{Q_{2}}$ and the linear projection $\pi: \mathbf{P}^{8--\rightarrow} \mathbf{P}^{5}$ given by $\pi:\left(z_{i j}\right) \mapsto\left(z_{0}, \ldots, z_{5}\right)=\left(z_{12}+z_{56}, z_{32}+z_{54}, z_{14}+z_{36}, 2 z_{34}, 2 z_{52}, 2 z_{16}\right)$. The center of the projection $\pi$ is easily seen to be disjoint from $\overline{\operatorname{im} \phi^{\prime}}$, so $\pi$ must map $\overline{\operatorname{im} \phi^{\prime}}$ to a four-fold in $\mathbf{P}^{5}$. In fact, this projection identifies points on $P_{1} \times P_{2} \subseteq \mathbf{P}^{8}$ via the involution which exchanges $P_{1}$ and $P_{2}, P_{1}$ and $P_{2}$ being identified via $\sigma^{7}$ acting on $\mathbf{P}_{-}^{5}$. One sees this by noting that the equations for $\pi$ are invariant under the induced action of $\sigma^{7}$ on $\mathbf{P}^{8}$. Now since $L$ has rank 1 on $\overline{\operatorname{im} \phi^{\prime}}$,

$$
L+L^{t}=\left(\begin{array}{ccc}
2 z_{16} & z_{14}+z_{36} & z_{12}+z_{56} \\
z_{14}+z_{36} & 2 z_{34} & z_{32}+z_{54} \\
z_{12}+z_{56} & z_{32}+z_{54} & 2 z_{52}
\end{array}\right)
$$

has rank $\leq 2$ on $\overline{\operatorname{im} \phi^{\prime}}$. Thus

$$
\operatorname{det}\left(\begin{array}{ccc}
z_{5} & z_{2} & z_{0} \\
z_{2} & z_{3} & z_{1} \\
z_{0} & z_{1} & z_{4}
\end{array}\right)=0
$$

is an equation for $\overline{\operatorname{im} \phi}$. As is well known (see for instance [28] or [1], pages 179180), this determines an irreducible cubic hypersurface which is the secant variety of the Veronese surface determined by the $2 \times 2$ minors of this matrix. Thus $\overline{\operatorname{im} \pi \circ \phi^{\prime}}$ must coincide with this cubic hypersurface, which is then isomorphic to $P_{1} \times P_{2} / \mathbf{Z}_{2}$. Since $Q_{2}$ can be expressed in terms of $z_{0}, \ldots, z_{5}$ as the Plücker quadric, $\pi \circ \phi^{\prime}\left(Q_{2}\right)$ is then the complete intersection of this Plücker quadric and the cubic hypersurface, showing (2).

To complete the proof of (3), note that $\pi: \overline{\operatorname{im} \phi^{\prime}} \rightarrow \overline{\operatorname{im} \pi \circ \phi^{\prime}}$ must be a $2: 1$ map. Thus the fibres of $\pi \circ \phi^{\prime}$ consist of pairs of lines. But in addition, one easily sees from the equations of $\phi$ and the structure of $\mathrm{GL}_{H}$ from Proposition 1.4 that 
the action of $\mathrm{GL}_{H}$ on $\mathbf{P}_{-}^{5}$ restricts to an action on the fibres of $\phi$, from which (3) follows.

(4) To prove that $Q_{2}$ is irreducible, first note that the inverse image of $\overline{\phi\left(Q_{2}\right)} \subseteq$ $P_{1} \times P_{2} / \mathbf{Z}_{2}$ in $P_{1} \times P_{2}$ is given by the intersection of the quadric

$$
2 z_{16}\left(z_{12}+z_{56}\right)-2 z_{52}\left(z_{32}+z_{54}\right)+2 z_{34}\left(z_{14}+z_{36}\right)=0
$$

and $P_{1} \times P_{2} \subseteq \mathbf{P}^{8}$. This coincides with $\overline{\phi^{\prime}\left(Q_{2}\right)}$. This divisor of type $(2,2)$ in $P_{1} \times P_{2}$ is easily seen to be irreducible by inspection. Now the proper transform of $Q_{2}$ in $X$ is a $\mathbf{P}^{1}$-bundle over $\overline{\phi^{\prime}\left(Q_{2}\right)}$, so $Q_{2}$ must be irreducible.

(5) By the above discussion, $\overline{\operatorname{im} \phi}$ is covered by $\overline{\phi^{\prime}\left(Q_{2}\right)}$, which is an irreducible divisor of type $(2,2)$ in $P_{1} \times P_{2}$. Thus $\overline{\operatorname{im} \phi}$ is irreducible. It is easy to see that an irreducible divisor $D$ in $P_{1} \times P_{2}$ of type $(2,2)$ is unirational: $D$ is a conic bundle via $p_{2}: P_{1} \times P_{2} \rightarrow P_{2}$, while if $l \subseteq P_{1}$ is a general line, then $p_{1}^{-1}(l) \cap D$ is a rational 2 -section of $p_{2}$, and thus $D$ is unirational; see e.g. [6], Cor. 4.4.

Theorem 3.2. $\overline{\operatorname{im} \phi}$ is birational to $\mathcal{A}_{14}^{H}$.

Proof. We know that $\overline{Z_{14}} \subseteq\left\{f_{1}=f_{2}=0\right\} \subseteq \mathbf{P}_{-}^{5}$. (We have not proven that the latter algebraic set is irreducible, so we don't know if this is an equality.) Let $x \in \overline{Z_{14}}$ be a general point, and consider the orbit $\mathrm{GL}_{H} \cdot x \subseteq \mathbf{P}_{-}^{5}$ of $x$. Since $x \in Q_{2}$, by Lemma $3.1(3), \mathrm{GL}_{H} \cdot x \subseteq Q_{2}$. Note that for general $x, \overline{\mathrm{GL}_{H} \cdot x}$ consists of two lines, say $l_{1}^{x} \cup l_{2}^{x}$. Where does $l_{1}^{x}$ intersect $\overline{Z_{14}}$ ? Since $\tau^{7} \in \mathrm{GL}_{H}$, both $x$ and $\tau^{7}(x) \in l_{1}^{x}$. In addition, if $T=\operatorname{diag}(i, 1, i, 1, \ldots)$, one sees that $T \in N(\mathcal{H}(1,14))$, so $T(x)$ and $\tau^{7}(T(x))$ are both in $l_{1}^{x} \cap \overline{Z_{14}}$. Furthermore, for general $x$, these four points are distinct. Similarly, $l_{2}^{x}=\sigma^{7}\left(l_{1}^{x}\right)$ intersects $\overline{Z_{14}}$ in at least four distinct points. If $l_{1}^{x}$ intersected $\overline{Z_{14}}$ in additional points, so would $l_{2}^{x}$, and vice versa, and then we would necessarily have $\overline{\mathrm{GL}_{X} \cdot x} \subseteq\left\{f_{1}=f_{2}=0\right\}$, which is a three-fold as $Q_{2}$ is irreducible, by Lemma 3.1. (4). Note that if $\overline{\mathrm{GL}_{H} \cdot x} \subseteq\left\{f_{1}=f_{2}=0\right\}$, then in particular, the fact that $f_{1}-f_{2}$ vanishes on the point $\left(a x_{1}, b x_{2}, a x_{3}, b x_{4}, a x_{5}, b x_{6}\right)$ for all $a$ and $b$ implies that $k_{1}:=x_{1} x_{3}^{3}+x_{1}^{3} x_{5}-x_{3} x_{5}^{3}=0$ and $k_{2}:=-x_{2}^{3} x_{4}+x_{4}^{3} x_{6}+x_{6}^{3} x_{2}=0$, i.e., $x$ is in the join of the Klein quartic curves in $P_{1}$ and $P_{2}$. Clearly $Q_{2}$ does not contain this join, and hence $x$ is contained in a surface $Q_{2} \cap\left\{k_{1}=k_{2}=0\right\}$, so $x$ is not a general point of $\overline{Z_{14}}$. Thus for general $x, l_{1}^{x}$ and $l_{2}^{x}$ intersect $\overline{Z_{14}}$ in precisely four points. In particular, $\overline{\mathrm{GL}_{H} \cdot x} \cap \overline{Z_{14}}$ is precisely the orbit of $x$ under the group $\mathrm{GL}_{H} \cap N(\mathcal{H}(1,14))$.

Thus, by Lemma $3.1(3), \overline{\phi\left(Z_{14}\right)}$ is birational to $\overline{Z_{14}} /\left(\mathrm{GL}_{H} \cap N(\mathcal{H}(1,14))\right)$, which in turn is birational to $\mathcal{A}_{14}^{H}$ by Theorem 1.6. But since $\overline{Z_{14}} \subseteq Q_{2}$ and $\overline{\phi\left(Z_{14}\right)} \subseteq \overline{\operatorname{im} \phi}$, and both of these latter varieties are irreducible three-folds, we must have equality, proving the theorem.

Remark 3.3. A more careful analysis of $\overline{\mathrm{im} \phi}$ in fact shows that this three-fold has a resolution of singularities which is a conic bundle over $\mathbf{P}^{2}$, with discriminant locus a non-singular plane curve of degree 6. It then follows from [6], Theorem 4.9, that this three-fold is irrational. Hence unirationality is the best we can achieve here.

Definition 3.4. Let $y \in \mathbf{P}_{-}^{5}$. Define $V_{14, y} \subseteq \mathbf{P}^{13}$ to be the scheme defined by the $4 \times 4$ Pfaffians of $M_{7}(x, y)$.

Also recall the definition of the Pfaffian Calabi-Yau three-fold in $\mathbf{P}^{6}$ from [17], $\S 5$. 
Definition 3.5. Let

$$
M_{7}^{\prime}(x, y)=\left(x_{3(i+j)} y_{3(i-j)}\right)_{i, j \in \mathbf{Z}_{7}}
$$

on $\mathbf{P}^{6} \times \mathbf{P}^{6}$. For $y \in \mathbf{P}_{-}^{2} \subseteq \mathbf{P}^{6}$, we define $V_{7, y}$ to be the scheme defined by the $6 \times 6$ Pfaffians of $M_{7}^{\prime}(x, y)$.

In [17], $\S 5, V_{7, y}$ was seen to be, for general $y$, a degree 14 Calabi-Yau three-fold with 49 ordinary double points, the latter being the $\mathbf{H}_{7}$-orbit of $y$.

$V_{14, y}$ is a linear section of $\operatorname{Gr}(2,7)$, and there is a correspondence between Pfaffian Calabi-Yau three-folds in $\mathbf{P}^{6}$ and such linear sections of $\operatorname{Gr}(2,7)$ in $\mathbf{P}^{13}$, which now appears to be folklore; see for example [26]. The general construction is as follows.

Let $V$ be a seven-dimensional vector space over $\mathbf{C}$, and let $W \subseteq \wedge^{2} V$ be a seven-dimensional subspace. Corresponding to this subspace is a subscheme $P f_{W}$ given by the intersection $\mathbf{P}(W) \cap D_{4} \subseteq \mathbf{P}\left(\bigwedge^{2} V\right)$, where $D_{4}$ is the projectivized locus of rank $\leq 4$ elements of $\bigwedge^{2} V$. This scheme $P f_{W}$ is, for general choice of $W$, a Pfaffian Calabi-Yau three-fold of degree 14 in $\mathbf{P}(W)$.

On the other hand, the annihilator $W^{0} \subseteq \bigwedge^{2} V^{\vee}$ of $W$ is a 14-dimensional subspace. Let $G r_{W^{0}}=\mathbf{P}\left(W^{0}\right) \cap D_{2}^{*} \subseteq \mathbf{P}\left(\bigwedge^{2} V^{\vee}\right)$, where $D_{2}^{*}$ is the locus of rank $\leq 2$ alternating forms on $V$. Of course $D_{2}^{*} \cong \operatorname{Gr}(2,7)$ via the Plücker embedding. For general $W, G r_{W^{0}}$ is a non-singular degree 42 Calabi-Yau three-fold in $\mathbf{P}^{13}$.

Remark 3.6. It has been observed in 26] that these two families of Calabi-Yau three-folds have the same mirror. This led us to conjecture in 1997 that $P f_{W}$ and $G r_{W^{0}}$ have isomorphic derived categories of coherent sheaves. This has now been proved by Borisov and Căldăraru [8] and Kuznetsov [21]. See also [18] for a physics argument, and the delightful term "glop" for this correspondence.

In what follows, $\mathbf{P}_{-}^{5}$ now denotes the negative eigenspace of $\iota$ acting on $\mathbf{P}^{13}$, and $\mathbf{P}_{-}^{2}$ denotes that of $\iota$ acting on $\mathbf{P}^{6}$.

Proposition 3.7. Let $\psi: \mathbf{P}_{-}^{5}{ }_{-\rightarrow} \mathbf{P}_{-}^{2}$ be defined by taking $x=\left(x_{1}, \ldots, x_{6}\right)$ to the kernel of the matrix

$$
\left(\begin{array}{lll}
x_{5} & x_{3} & x_{1} \\
x_{2} & x_{4} & x_{6}
\end{array}\right)
$$

i.e.,

$$
\psi\left(x_{1}, \ldots, x_{6}\right)=\left(x_{3} x_{6}-x_{1} x_{4}, x_{1} x_{2}-x_{5} x_{6}, x_{4} x_{5}-x_{2} x_{3}\right) .
$$

Then

(1) The fibres of $\psi$ are $\mathbf{P}^{3}$ 's which are closures of $G L^{2 K(1,14)}$-orbits.

(2) Under the correspondence between Pfaffian and Grassmann Calabi-Yau three-folds, $V_{14, y}$ corresponds to $V_{7, \psi(y)}$.

Proof. (1) follows easily from the description of $G L^{2 K(1,14)}$ in Proposition 1.4

(2) Let $y^{\prime}=\left(y_{0}^{\prime}, \ldots, y_{6}^{\prime}\right) \in \mathbf{P}_{-}^{2} \subseteq \mathbf{P}^{6}=\mathbf{P}(V)$, with $y_{i}^{\prime}=-y_{-i}^{\prime}$. Then $V_{7, y^{\prime}}$ is given by the ideal generated by $6 \times 6$ Pfaffians of $M_{7}^{\prime}\left(x, y^{\prime}\right)$. Let $z_{i, j}$ be coordinates on $\mathbf{P}^{20}=\mathbf{P}\left(\bigwedge^{2} V\right)$, with $0 \leq i, j \leq 6, z_{i, j}=-z_{j, i}$, so that $\left(z_{i, j}\right)_{0 \leq i, j \leq 6}$ is the corresponding skew-symmetric matrix. Then $M_{7}^{\prime}\left(x, y^{\prime}\right)$ defines a map $\alpha: \mathbf{P}^{6} \rightarrow \mathbf{P}^{20}$ via

$$
z_{i, j}=x_{3(i+j)} y_{3(i-j)}^{\prime}
$$

Now $\alpha$ is, for general choice of $y^{\prime}$, an embedding, and $V_{7, y^{\prime}}=\alpha^{-1}\left(D_{4}\right)$. 
Now we wish to compute the equations of the annihilator of $\alpha\left(\mathbf{P}^{6}\right)$ in $\mathbf{P}\left(\bigwedge^{2} V^{\vee}\right)$. Note that $\alpha\left(\mathbf{P}^{6}\right)$ is spanned by the images of $e_{0}, \ldots, e_{6}$ (the standard basis vectors), and $e_{i}$ is mapped via $\alpha$ to the point with coordinates

$$
z_{j, k}=\delta_{i, 3(j+k)} y_{3(j-k)}^{\prime} .
$$

The ideal of the annihilator is generated by the hyperplanes in $\mathbf{P}\left(\bigwedge^{2} V^{\vee}\right)$ corresponding to these seven points. If $z_{i, j}^{*}$ are the dual coordinates on $\mathbf{P}\left(\bigwedge^{2} V^{\vee}\right)$, then this gives us the ideal for the annihilator generated by the seven equations

$$
y_{1}^{\prime} z_{1+i, 6+i}^{*}+y_{2}^{\prime} z_{2+i, 5+i}^{*}+y_{3}^{\prime} z_{3+i, 4+i}^{*}=0, \quad 0 \leq i \leq 6 .
$$

This defines $L \subseteq \mathbf{P}\left(\bigwedge^{2} V^{\vee}\right)$ and $L \cong \mathbf{P}^{13}$, and $L \cap D_{2}^{*}$ is the Grassmann Calabi-Yau corresponding to $V_{7, y^{\prime}}$. To compare this with $V_{14, y}$ for some $y$, we have to find a parametrization $\beta: \mathbf{P}^{13} \rightarrow L$ given by the matrix

$$
M_{7}(x, y)=\left(x_{i+j} y_{i-j}+x_{i+j+7} y_{i-j+7}\right)_{0 \leq i, j \leq 6} .
$$

In other words, a point $y \in \mathbf{P}_{-}^{5}$ yields a linear map $\beta: \mathbf{P}^{13} \rightarrow \mathbf{P}\left(\bigwedge^{2} V^{\vee}\right)$ by taking the point $x=\left(x_{i}\right) \in \mathbf{P}^{13}$ to the point with coordinates $\left(z_{i, j}^{*}\right)$ given by

$$
z_{i, j}^{*}=x_{i+j} y_{i-j}+x_{i+j+7} y_{i-j+7}, \quad 0 \leq i, j \leq 6,
$$

and $V_{14, y}=G r_{\mathrm{im} \beta}$. In order for the image of $\beta$ to be $L$, the seven equations defining $L$ must be satisfied by any point in the image of $\beta$, i.e.,

$$
y_{1}^{\prime}\left(x_{7+2 i} y_{9}+x_{2 i} y_{2}\right)+y_{2}^{\prime}\left(x_{7+2 i} y_{11}+x_{2 i} y_{4}\right)+y_{3}^{\prime}\left(x_{7+2 i} y_{13}+x_{2 i} y_{6}\right)=0
$$

for $0 \leq i \leq 6$. Since this must hold for all values of $x$, and keeping in mind in addition that $y \in \mathbf{P}_{-}^{5}$, we find we must have

$$
\begin{aligned}
& y_{1}^{\prime} y_{5}+y_{2}^{\prime} y_{3}+y_{3}^{\prime} y_{1}=0 \\
& y_{1}^{\prime} y_{2}+y_{2}^{\prime} y_{4}+y_{3}^{\prime} y_{6}=0 .
\end{aligned}
$$

This is precisely equivalent to the statement

$$
\left(y_{1}^{\prime}, y_{2}^{\prime}, y_{3}^{\prime}\right) \in \operatorname{ker}\left(\begin{array}{lll}
y_{5} & y_{3} & y_{1} \\
y_{2} & y_{4} & y_{6}
\end{array}\right)
$$

In other words, $y^{\prime}=\psi(y)$. It is also easy to see that the map $\beta$ defined by such a choice of $y$ is an isomorphism with $L$ as long as $\left(y_{5}, y_{3}, y_{1}\right)$ and $\left(y_{2}, y_{4}, y_{6}\right)$ are linearly independent.

Remark 3.8. Using Macaulay or Macaulay 2 [5], 10, one can check the following three facts:

(1) For general $y \in \mathbf{P}_{-}^{5}, V_{14, y}$ contains a pencil of $(1,14)$-polarized abelian surfaces invariant under the subgroup $\left\langle\sigma^{2}, \tau^{2}\right\rangle$ of $\mathbf{H}_{14}$.

(2) For general $y \in \mathbf{P}_{-}^{5}, V_{14, y}$ is an irreducible three-fold of degree 42 with 49 ordinary double points.

(3) If $y \in Z_{14}$ is general, then the $6 \times 6$ Pfaffians of $M_{7}\left(x, \sigma^{7}(y)\right)$ induce a birational map $V_{14, y^{--} \rightarrow} V_{7, \psi(y)}$.

Unfortunately, we do not know a non-computational proof of (2) and (3). It is intriguing that the correspondence between $P f_{W}$ and $G r_{W^{0}}$ identifies, in the particular case of $V_{14, y}$ and $V_{7, \psi(y)}$, birational Calabi-Yau three-folds. This is not true in general: it is easy to see that for general $W, P f_{W}$ and $G r_{W^{0}}$ are not 
birationally equivalent, as they both have Picard number one, but for $H$ a primitive generator of the Picard group, $H^{3}=14$ and 42 , respectively.

Note that (3) implies that the Calabi-Yau $V_{14, y}$, or equivalently, $V_{7, \psi(y)}$, is birationally fibred by abelian surfaces in two different ways: one fibration having $(1,7)$ polarized fibres and one having $(1,14)$-polarized fibres. The existence of these two distinct pencils of abelian surfaces was observed in Remark 2.9 of [23].

\section{Moduli of $(1,16)$-POlarized abelian surfaces}

Restricting $M_{8}(x, x)$ to $\mathbf{P}_{-}^{6}$, one finds a $5 \times 5$ block

$$
M=\left(\begin{array}{ccccc}
0 & -x_{1}^{2}-x_{7}^{2} & -x_{2}^{2}-x_{6}^{2} & -x_{3}^{2}-x_{5}^{2} & -2 x_{4}^{2} \\
x_{1}^{2}+x_{7}^{2} & 0 & -x_{1} x_{3}-x_{5} x_{7} & -x_{2} x_{4}-x_{4} x_{6} & -2 x_{3} x_{5} \\
x_{2}^{2}+x_{6}^{2} & x_{1} x_{3}+x_{5} x_{7} & 0 & -x_{1} x_{5}-x_{3} x_{7} & -2 x_{2} x_{6} \\
x_{3}^{2}+x_{5}^{2} & x_{2} x_{4}+x_{4} x_{6} & x_{1} x_{5}+x_{3} x_{7} & 0 & -2 x_{1} x_{7} \\
2 x_{4}^{2} & 2 x_{3} x_{5} & 2 x_{2} x_{6} & 2 x_{1} x_{7} & 0
\end{array}\right) .
$$

In the discussion that follows, it will be helpful to make a change of coordinates. We define a new set of coordinates $\left(y_{0}, y_{1}, z_{0}, z_{1}, t_{0}, t_{1}, u\right)$ on $\mathbf{P}_{-}^{6}$ by

$y_{0}=x_{1}+x_{7}, y_{1}=x_{3}+x_{5}, z_{0}=x_{1}-x_{7}, z_{1}=x_{3}-x_{5}, t_{0}=x_{4}, t_{1}=x_{2}+x_{6}, u=x_{2}-x_{6}$.

In these coordinates,

$$
M=\frac{1}{2}\left(\begin{array}{ccccc}
0 & -y_{0}^{2}-z_{0}^{2} & -t_{1}^{2}-u^{2} & -y_{1}^{2}-z_{1}^{2} & -4 t_{0}^{2} \\
y_{0}^{2}+z_{0}^{2} & 0 & -y_{0} y_{1}-z_{0} z_{1} & -2 t_{0} t_{1} & -y_{1}^{2}+z_{1}^{2} \\
t_{1}^{2}+u^{2} & y_{0} y_{1}+z_{0} z_{1} & 0 & -y_{0} y_{1}+z_{0} z_{1} & -t_{1}^{2}+u^{2} \\
y_{1}^{2}+z_{1}^{2} & 2 t_{0} t_{1} & y_{0} y_{1}-z_{0} z_{1} & 0 & -y_{0}^{2}+z_{0}^{2} \\
4 t_{0}^{2} & y_{1}^{2}-z_{1}^{2} & t_{1}^{2}-u^{2} & y_{0}^{2}-z_{0}^{2} & 0
\end{array}\right) .
$$

Let $Z \subseteq \mathbf{P}_{-}^{6}$ be the subvariety defined by the $4 \times 4$ Pfaffians of $M$. Clearly $\overline{Z_{16}} \subseteq Z$.

Lemma 4.1. $Z$ is an irreducible three-fold of degree 40 in $\mathbf{P}_{-}^{6}$. In particular $\overline{Z_{16}}=Z$.

Proof. If $Z$ is in fact a three-fold, then $Z$ is of the expected degree for such a Pfaffian variety, and standard degree calculations show it must be of degree 40 . To show it is an irreducible three-fold, we must work harder. First, consider the point

$$
p=\left(y_{0}, y_{1}, z_{0}, z_{1}, t_{0}, t_{1}, u\right)=(0,0,0,0,0,0,1) \in \mathbf{P}_{-}^{6} .
$$

The tangent cone to $Z$ at $p$ can be computed by working on the affine open set of $\mathbf{P}_{-}^{6}$ where $u=1$ and by identifying the leading terms of the $4 \times 4$ Pfaffians of $M$ with $u$ replaced by 1 . These leading terms yield the following equations for the tangent cone at $p$ :

$$
2 t_{0} t_{1}=y_{0}^{2}-z_{0}^{2}+y_{1}^{2}+z_{1}^{2}=y_{0}^{4}-y_{1}^{4}-z_{0}^{4}+z_{1}^{4}+8 t_{0}^{3} t_{1}=y_{0}^{2}+y_{1}^{2}+z_{0}^{2}-z_{1}^{2}=0 .
$$

Note however that $y_{0}^{4}-y_{1}^{4}-z_{0}^{4}+z_{1}^{4}+8 t_{0}^{3} t_{1}$ is in the ideal generated by the three quadrics, so in fact the tangent cone has ideal

$$
I=\left(t_{0} t_{1}, y_{0}^{2}+y_{1}^{2}, z_{0}^{2}-z_{1}^{2}\right) .
$$

This is in fact a union of 8 affine 3 -planes.

Now consider the projection map $\pi: \mathbf{P}_{-}^{6}-\rightarrow \mathbf{P}^{5}$ given by $\left(y_{0}, y_{1}, z_{0}, z_{1}, t_{0}, t_{1}, u\right) \mapsto$ $\left(y_{0}, y_{1}, z_{0}, z_{1}, t_{0}, t_{1}\right)$ and its restriction $\pi: Z-\rightarrow \mathbf{P}^{5}$. First note that $\pi$ does not map any component of $Z$ to something of lower dimension. If it did, the component 
would have to be a cone with vertex at $p$. Such a component would then appear as a component of the tangent cone. However, one easily checks that none of the 8 three-planes in the tangent cone at $p$ are in fact contained in $Z$. To understand the image $\pi(Z)$, we first find two equations vanishing on $\pi(Z)$. Note that the Pfaffian of the submatrix of $M$ obtained by deleting the third row and third column of $M$ does not depend on $u$, and this Pfaffian vanishes on $\pi(Z)$. It is

$$
g_{1}=y_{0}^{4}-y_{1}^{4}-z_{0}^{4}+z_{1}^{4}+8 t_{0}^{3} t_{1} .
$$

For another equation, one adds the Pfaffian obtained by deleting the first row and column of $M$ with the Pfaffian obtained by deleting the fifth row and fifth column to get

$$
g_{2}=2 y_{0}^{3} y_{1}+2 y_{0} y_{1}^{3}-2 z_{0}^{3} z_{1}+2 z_{0} z_{1}^{3}-4 t_{0} t_{1}^{3} .
$$

Thus $\pi(Z) \subseteq Z^{\prime}=\left\{g_{1}=g_{2}=0\right\}$. Now the singular locus of $Z^{\prime}$ can be computed by hand, and one finds in fact that $Z^{\prime}$ is a codimension 2 complete intersection (hence of degree 16) with 6 singular points at $(1, \pm i, 0,0,0,0),(0,0,1, \pm 1,0,0)$, $(0,0,0,0,1,0)$, and $(0,0,0,0,0,1)$. Thus in particular $Z^{\prime}$ is irreducible, so $\pi(Z)=$ $Z^{\prime}$. We also see that $\pi$ maps $Z$ two-to-one to $Z^{\prime}$, to account for the degree of $Z^{\prime}$ : $\operatorname{deg} Z^{\prime}=\left(\operatorname{deg} Z-\operatorname{mult}_{P} Z\right) / \operatorname{deg} \pi$. In fact, $Z$ is clearly invariant under the involution $j: \mathbf{P}_{-}^{6} \rightarrow \mathbf{P}_{-}^{6}$ given by negation of the coordinate $u$, and this interchanges the two sheets of the double covering $\pi: Z--\rightarrow Z^{\prime}$. Thus $Z$ consists of either one or two irreducible components. If it is the latter, then $j$ interchanges these two components. Note that in coordinates $x_{1}, \ldots, x_{7}, j$ acts by interchanging $x_{2}$ and $x_{6}$.

To show that $Z$ is irreducible, we note that if not, then the branch locus $B \subseteq Z^{\prime}$ of the double covering $p: Z--\rightarrow Z^{\prime}$ must be non-reduced everywhere. This branch locus is the image of $\operatorname{Fix}(j) \cap Z$, which is easily computed by setting $u=0$ in the matrix $M$ and taking $4 \times 4$ Pfaffians to obtain the equations for $B \subseteq Z^{\prime}$. We then just need to identify one point in $B$ where the tangent cone to $B$ is reduced. Such a point is $\left(y_{0}, \ldots, t_{1}\right)=(0,0,0,0,0,1)$, which is contained in $B$ and whose tangent cone is easily seen, as we saw earlier, to be given by

$$
t_{0}=y_{0}^{2}-y_{1}^{2}=z_{0}^{2}+z_{1}^{2}=0
$$

which is reduced.

Remark 4.2. With a bit of additional calculation, one finds that the 6 singularities of $Z^{\prime} \subseteq \mathbf{P}^{5}$ are ordinary triple points (i.e. having tangent cone be a cone over a non-singular cubic surface). In particular these are canonical singularities. Thus $Z^{\prime}$ is in fact of general type with canonical bundle $\mathcal{O}_{Z^{\prime}}(2)$. Thus $Z=\overline{Z_{16}}$ is also of general type.

Our goal now is to understand $\mathcal{A}_{16}^{H}$ with $H=2 K(1,16)$. In this case, $\mathrm{GL}_{H}$ is a four-dimensional group, as computed in Proposition 1.4. First, we will understand the set of three-dimensional $\mathrm{GL}_{H}$ orbits in $\mathbf{P}_{-}^{6}$.

For $x=\left(x_{1}, \ldots, x_{7}\right) \in \mathbf{P}_{-}^{6}$, let $N_{x}$ be the matrix

$$
N_{x}=\left(\begin{array}{ccccccc}
-z_{1} & 0 & z_{0} & 0 & -z_{0} & 0 & z_{1} \\
0 & t_{0} & 0 & -t_{1} & 0 & t_{0} & 0 \\
y_{1} & 0 & -y_{0} & 0 & -y_{0} & 0 & y_{1}
\end{array}\right)
$$

We can then define a rational map $\phi: \mathbf{P}_{-}^{6}{ }_{--} \rightarrow \operatorname{Gr}(4,7)$ by $x \mapsto \operatorname{ker} N_{x}$. Here the columns of $N_{x}$ correspond to the coordinates $x_{1}, \ldots, x_{7}$. 
Lemma 4.3. (1) $\operatorname{rank}\left(N_{x}\right)<3$ if and only if $x \in \Pi_{1} \cup \Pi_{2} \cup \Pi_{3}$, where

$$
\begin{aligned}
& \Pi_{1}=\left\{z_{0}=z_{1}=0\right\}, \\
& \Pi_{2}=\left\{t_{0}=t_{1}=0\right\}, \\
& \Pi_{3}=\left\{y_{0}=y_{1}=0\right\} .
\end{aligned}
$$

(2) $\operatorname{dim} \overline{\mathrm{GL}_{H} \cdot x}=3$ if and only if $x \notin \Pi_{1} \cup \Pi_{2} \cup \Pi_{3} \cup\{u=0\}$.

(3) $\overline{\mathrm{GL}_{H} \cdot x}=\mathbf{P}\left(\operatorname{ker} N_{x}\right)$ if $x \notin \Pi_{1} \cup \Pi_{2} \cup \Pi_{3} \cup\{u=0\}$.

(4) $\overline{\operatorname{im} \phi}$ is birational to $\mathbf{P}^{1} \times \mathbf{P}^{1} \times \mathbf{P}^{1}$.

Proof. (1) Clearly $N_{x}$ drops rank on $\Pi_{1} \cup \Pi_{2} \cup \Pi_{3}$. Conversely, if $N_{x}$ is rank $\leq 2$, then either the second row of $N_{x}$ is zero or the first and third rows are linearly dependent. This latter case occurs only on $\Pi_{1} \cup \Pi_{3}$.

(2) By Proposition 1.4, one sees that the action of $\alpha \in \mathrm{GL}_{H}$ on $\mathbf{P}_{-}^{6}$ is of the form

$$
\begin{aligned}
\alpha:\left(x_{1}, \ldots, x_{7}\right) \mapsto & \left(a x_{1}-b x_{7}, c x_{2}-d x_{6},\right. \\
& a x_{3}-b x_{5},(c-d) x_{4}, a x_{5}-b x_{3}, \\
& \left.c x_{6}-d x_{2}, a x_{7}-b x_{1}\right)
\end{aligned}
$$

for suitable $a, b, c, d \in \mathbf{C}$. In our new coordinates this is

$$
\begin{aligned}
& \alpha:\left(y_{0}, y_{1}, z_{0}, z_{1}, t_{0}, t_{1}, u\right) \mapsto \\
& \quad\left((a-b) y_{0},(a-b) y_{1},(a+b) z_{0},(a+b) z_{1},(c-d) t_{0},(c-d) t_{1},(c+d) u\right) .
\end{aligned}
$$

Thus $\mathrm{GL}_{H} \cong\left(\mathbf{C}^{*}\right)^{4}$, and $\operatorname{dim} \overline{\mathrm{GL}_{H} \cdot x}<3$ if and only if $x$ is in $\Pi_{1}, \Pi_{2}, \Pi_{3}$, or $\{u=0\}$. (2) then follows.

(3) We first note that $N_{x} \cdot x=0$, so $x$ is in the $\mathbf{P}^{3}$ represented by $\phi(x)$. From the form of $\alpha$ given in (2), one sees that $N_{\alpha(x)}$ is obtained from $N_{x}$ by multiplying the first row of $N_{x}$ by $a+b$, the second row by $c-d$ and the third by $a-b$. Thus as $N_{\alpha(x)} \cdot \alpha(x)=0$, we also have $N_{x} \cdot \alpha(x)=0$, so $\overline{\mathrm{GL}_{H} \cdot x} \subseteq \mathbf{P}\left(\operatorname{ker} N_{x}\right)$. By (1) and (2), these are the same dimension, so equality holds.

(4) Let $L_{1}, L_{2}, L_{3} \subseteq \mathbf{P}_{-}^{6}{ }^{\vee}$ be lines dual to $\Pi_{1}, \Pi_{2}$ and $\Pi_{3}$, respectively. If $p \in \operatorname{im} \phi$, then $p$ corresponds to a $\mathbf{P}^{3} \subseteq \mathbf{P}_{-}^{6}$, with dual $\mathbf{P}^{2}$ being, say, $P \subseteq \mathbf{P}_{-}^{6}$. Then $P \cap L_{i}$ consists of one point for each $i$. This gives a birational map between $\overline{\operatorname{im} \phi}$ and $L_{1} \times L_{2} \times L_{3}$.

\section{Lemma 4.4 .}

$$
\#\left(\frac{\mathrm{GL}_{H} \cap N(\mathcal{H}(1,16))}{\mathbf{C}^{*}}\right)=32 .
$$

Proof. First, $G L_{H} \cap \mathcal{H}(1,16)$ is generated by $\mathbf{C}^{*}, \sigma^{8}$ and $\tau^{8}$, as is easily seen from Proposition 1.4. Taking the quotient of $N(\mathcal{H}(1,16))$ by $\mathcal{H}(1,16)$ gives $S L_{2}\left(\mathbf{Z}_{16}\right)$. Thus it is enough to identify the image of $G L_{H} \cap N(\mathcal{H}(1,16))$ in $S L_{2}\left(\mathbf{Z}_{16}\right)$; i.e., as in the proof of Proposition [1.2, we just need to identify the subgroup of $S L_{2}\left(\mathbf{Z}_{16}\right)$, keeping $2 K(1,16)$ invariant. These are the elements $\alpha$ of $S L_{2}\left(\mathbf{Z}_{16}\right), \alpha=\left(\alpha_{i j}\right)_{1 \leq i, j \leq 2}$, with $\alpha(2,0)=(2,0), \alpha(0,2)=(0,2)$. Thus $\alpha_{11}$ and $\alpha_{22}$ are either 1 or 9 , and $\alpha_{12}$ and $\alpha_{21}$ are either 0 or 8 . This gives a total of 16 possibilities; but of these, half have determinant 9 , leaving a total of 8 such elements with determinant 1 . Since $\#\left(G L_{H} \cap \mathcal{H}(1,16)\right) / \mathbf{C}^{*}=4$, this gives a total of $4 \times 8=32$, as desired. 
Theorem 4.5. $\overline{\operatorname{im} \phi}$ is birational to $\mathcal{A}_{16}^{H}$.

Proof. Lemma 4.3 shows that $\left(\mathbf{P}_{-}^{6} \backslash\left(\Pi_{1} \cup \Pi_{2} \cup \Pi_{3} \cup\{u=0\}\right)\right) / \mathrm{GL}_{H}$ is birational to $\mathbf{P}^{1} \times \mathbf{P}^{1} \times \mathbf{P}^{1}$ via the map $\phi$. On the other hand, by Theorem 1.6. $\mathcal{A}_{16}^{H}$ is birational to $Z_{16} / \mathrm{GL}_{H} \cap N(\mathcal{H}(1,16))$. Thus to show $\mathcal{A}_{16}^{H}$ is birational to $\mathbf{P}^{1} \times$ $\mathbf{P}^{1} \times \mathbf{P}^{1}$, it is enough to show that for a general point $x \in Z_{16},\left(\mathrm{GL}_{H} \cdot x\right) \cap Z_{16}=$ $\left(\mathrm{GL}_{H} \cap N(\mathcal{H}(1,16))\right) \cdot x$. By Lemma 4.4, it is enough then to show that \# $\left(\mathrm{GL}_{H} \cdot x\right) \cap$ $Z_{16}=32$ for $x \in Z_{16}$ a general point. Since $\left(\mathrm{GL}_{H} \cap N(\mathcal{H}(1,16))\right) \cdot x \subseteq\left(\mathrm{GL}_{H} \cdot x\right) \cap$ $Z_{16}$, clearly $\#\left(\mathrm{GL}_{H} \cdot x\right) \cap Z_{16} \geq 32$. On the other hand, $\overline{Z_{16}}$ is degree 40 , so if $\overline{\mathrm{GL}_{H} \cdot x} \cap \overline{Z_{16}}$ is a finite set of points, we must then have

$$
\#\left(\mathrm{GL}_{H} \cdot x\right) \cap Z_{16} \leq 40 .
$$

Since this number must be divisible by 32 , we see that $\#\left(\mathrm{GL}_{H} \cdot x\right) \cap Z_{16}=32$. Thus we are done if we can find a point $x \in Z_{16}$ such that $\overline{\mathrm{GL}_{H} \cdot x} \cap \overline{Z_{16}}$ is finite. Since the variety $\overline{Z_{16}}$ is defined over the ring $\mathbf{Z}[1 / 2]$, it is enough to find such a point in finite odd characteristic. We know of no better way of finding such a point except by searching exhaustively. For example, in characteristic $p=7$, one finds the point with coordinates $y_{0}=4, y_{1}=-1, z_{0}=-2, z_{1}=-1, t_{0}=2, t_{1}=-2, u=1$, does the trick. This can be checked in Macaulay/Macaulay 2.

\section{Moduli of $(1,18)$-POLARIZED ABELIAN SURFACES}

Again, we study $\mathcal{A}_{18}^{H}$ with $H=2 K(1,18)$. As we shall see, the closure of $\mathrm{GL}_{H} \cdot Z_{18}$ is a quartic hypersurface in $\mathbf{P}_{-}^{7}$, and we can use similar tricks to the $(1,14)$ case to understand the correct quotient of $\overline{Z_{18}}$.

The matrix $M_{9}\left(\sigma \tau^{9}(x), x\right)$ restricted to $\mathbf{P}_{-}^{7}$ has a $4 \times 4$ block obtained by taking the $i j$ th entries with $1 \leq i \leq 4,1 \leq j \leq 4$, of the form

$$
M^{\prime}:=\left(\begin{array}{cccc}
0 & x_{1} x_{2}-x_{7} x_{8} & -x_{2} x_{3}+x_{6} x_{7} & x_{3} x_{4}-x_{5} x_{6} \\
-x_{1} x_{2}+x_{7} x_{8} & 0 & x_{1} x_{4}-x_{5} x_{8} & -x_{2} x_{5}+x_{4} x_{7} \\
x_{2} x_{3}-x_{6} x_{7} & -x_{1} x_{4}+x_{5} x_{8} & 0 & x_{1} x_{6}-x_{3} x_{8} \\
-x_{3} x_{4}+x_{5} x_{6} & x_{2} x_{5}-x_{4} x_{7} & -x_{1} x_{6}+x_{3} x_{8} & 0
\end{array}\right) .
$$

Let $f$ be the Pfaffian of $M^{\prime}$ and let $Q=\{f=0\}$. We also define a rational map $\phi: \mathbf{P}_{-}^{7}-\rightarrow \operatorname{Gr}(2,4)$ by taking a point $\left(x_{1}, \ldots, x_{8}\right)$ to the subspace of $\mathbf{C}^{4}$ spanned by the rows of the matrix

$$
N=\left(\begin{array}{llll}
x_{1} & x_{3} & x_{5} & x_{7} \\
x_{8} & x_{6} & x_{4} & x_{2}
\end{array}\right)
$$

Explicitly in Plücker coordinates,

$$
\begin{aligned}
\left(x_{1}, \ldots, x_{8}\right) \mapsto\left(y_{0}, \ldots, y_{5}\right)= & \left(x_{1} x_{6}-x_{3} x_{8},-x_{1} x_{4}+x_{5} x_{8}, x_{1} x_{2}-x_{7} x_{8}, x_{3} x_{4}-x_{5} x_{6},\right. \\
& \left.-x_{2} x_{3}+x_{6} x_{7}, x_{2} x_{5}-x_{4} x_{7}\right) .
\end{aligned}
$$

Lemma 5.1. (1) $\phi$ fails to be defined precisely on the fourfold $Z \subseteq \mathbf{P}_{-}^{7}$ defined by the $2 \times 2$ minors of $N$; this is isomorphic to $\mathbf{P}^{1} \times \mathbf{P}^{3}$.

(2) If $X$ is the blow-up of $\mathbf{P}_{-}^{7}$ along $Z$, then $\phi$ lifts to a morphism $\tilde{\phi}: X \rightarrow$ $\operatorname{Gr}(2,4)$ which is a surjection, a $\mathbf{P}^{3}$ bundle, and each fibre of $\tilde{\phi}$ is the closure of $a \mathrm{GL}_{H}$ orbit. 
(3) If $\tilde{Q}$ is the proper transform of $Q$, then $\tilde{\phi}(\tilde{Q})$ is a $(2,2)$ complete intersection defined by the equations

$$
\begin{aligned}
& y_{0} y_{5}-y_{1} y_{4}+y_{2} y_{3}=0, \\
& y_{0} y_{2}-y_{1} y_{3}+y_{4} y_{5}=0
\end{aligned}
$$

and $\tilde{Q}$ is a $\mathbf{P}^{3}$-bundle over $\tilde{\phi}(\tilde{Q})$ whose fibres are all closures of $\mathrm{GL}_{H}$-orbits.

(4) $\tilde{\phi}(\tilde{Q})$ is rational.

Proof. (1) is standard. For (2), $\phi$ clearly lifts to the blowup, $\tilde{\phi}: X \rightarrow \operatorname{Gr}(2,4)$. It is a surjection since any two-dimensional subspace of $\mathbf{C}^{4}$ can be expressed as the row span of some $2 \times 4$ matrix. Now an element $T=\left(a_{i j}\right) \in G L_{H}$ is determined by $\alpha=a_{00}, \beta=a_{11}, \gamma=a_{0 d}$ and $\delta=a_{1, d+1}$, by Proposition 1.4. (2), and restricting the action of $T$ to $\mathbf{P}_{-}^{7}$, one sees that the matrix $N$ is transformed by $T$ to

$$
\left(\begin{array}{cccc}
\beta x_{1}-\delta x_{8} & \beta x_{3}-\delta x_{6} & \beta x_{5}-\delta x_{4} & \beta x_{7}-\delta x_{2} \\
\alpha x_{8}-\gamma x_{1} & \alpha x_{6}-\gamma x_{3} & \alpha x_{4}-\gamma x_{5} & \alpha x_{2}-\gamma x_{7}
\end{array}\right),
$$

which clearly has the same row span as $N$. So fibres of $\tilde{\phi}$ are $\mathrm{GL}_{H}$ invariant. In fact, two rank two $2 \times 4$ matrices $N$ and $N^{\prime}$ have the same row span if and only if $N^{\prime}$ is obtained from $N$ in this fashion, for some choice of $\alpha, \beta, \gamma, \delta$, necessarily with $\alpha \beta-\gamma \delta \neq 0$. So the fibres of $\tilde{\phi}$ are always closures of $\mathrm{GL}_{H}$ orbits. The closure of the $\mathrm{GL}_{H}$ orbit of $\left(x_{1}, \ldots, x_{8}\right)$ is then the span of the points $\left(x_{1}, 0, x_{3}, 0, x_{5}, 0, x_{7}, 0\right)$, $\left(x_{8}, 0, x_{6}, 0, x_{4}, 0, x_{2}, 0\right),\left(0, x_{2}, 0, x_{4}, 0, x_{6}, 0, x_{8}\right)$ and $\left(0, x_{7}, 0, x_{5}, 0, x_{3}, 0, x_{1}\right)$. Hence this is a $\mathbf{P}^{3}$ as long as this orbit is three-dimensional, which is the case if $\left(x_{1}, \ldots, x_{8}\right)$ $\notin Z$. This shows $(2)$.

(3) The first quadric is the Plücker relation defining $\operatorname{Gr}(2,4)$, and the second equation just pulls back to the equation $f$. The singularities of this complete intersection are easily computed and found to consist of 6 points; hence it must be irreducible. Now the fibres of $\left.\tilde{\phi}\right|_{\tilde{Q}}$ are dimension $\leq 3$, and $\tilde{Q}$ is dimension 6 , so in fact the fibres of $\left.\tilde{\phi}\right|_{\tilde{Q}}$ are three-dimensional and $\tilde{\phi}(\tilde{Q})$ is three-dimensional. Hence $\tilde{\phi}(\tilde{Q})$ is precisely the complete intersection and the fibres are $\mathbf{P}^{3}$ 's.

(4) One easily checks that the point $P=\left(y_{0}, \ldots, y_{5}\right)=(1,0,0,1,1,0)$ is a double point. Indeed, setting $y_{0}=1$ and expanding the two equations in Taylor series shows the tangent cone to $\tilde{\phi}(\tilde{Q})$ at $P$ is a quadric cone. Consider the projection from $P$ given by

$$
\left(y_{0}, \ldots, y_{5}\right) \mapsto\left(z_{1}, \ldots, z_{5}\right)=\left(y_{1}, y_{2}, y_{3}-y_{0}, y_{4}-y_{0}, y_{5}\right) .
$$

Noting that the difference of the two quadratic equations defining $\tilde{\phi}(\tilde{Q})$ is

$$
-y_{1}\left(y_{4}-y_{3}\right)+y_{2}\left(y_{3}-y_{0}\right)+y_{5}\left(y_{0}-y_{4}\right)=-z_{1}\left(z_{4}-z_{3}\right)+z_{2} z_{3}-z_{5} z_{4},
$$

which defines a quadric cone $Q^{\prime}$ in $\mathbf{P}^{4}$ with one singular point, we see that the image of $\tilde{\phi}(\tilde{Q})$ under this projection is contained in $Q^{\prime}$. Since $\tilde{\phi}(\tilde{Q})$ is degree 4 , there are two possibilities. Either the projection $\tilde{\phi}(\tilde{Q})^{--\rightarrow} Q^{\prime}$ is birational or $\tilde{\phi}(\tilde{Q})$ is a cone with vertex $P$. The latter is impossible, since as observed earlier, $\tilde{\phi}(\tilde{Q})$ has 6 isolated singular points.

We next need to refine the results of [15]:

Proposition 5.2. For a general $\mathbf{H}_{18}$-invariant $(1,18)$-polarized abelian surface $A \subseteq$ $\mathbf{P}^{17}$ and a point $y \in A \cap \mathbf{P}_{-}^{7}$, the $4 \times 4$ Pfaffians of the matrix $M_{9}\left(\sigma \tau^{9}(x), y\right)$ generate the homogeneous ideal of $A$. 
Proof. As in the proof of [15, Theorem 6.2, it is enough to show there is a point $y \in \overline{Z_{18}}$ such that the $4 \times 4$ Pfaffians of the matrix $M\left(\sigma \tau^{9}(x), y\right)$ generate the homogeneous ideal of a surface $S$ which has the same Hilbert polynomial as a $(1,18)$-polarized abelian surface in $\mathbf{P}^{17}$. We proceed as follows to find such a point.

Let $E_{1} \subseteq \mathbf{P}^{5}$ be a general $\mathbf{H}_{6}$-invariant elliptic curve and $E_{2} \subseteq \mathbf{P}^{17}$ be an $\mathbf{H}_{18}$-invariant elliptic curve; thus $E_{1}$ and $E_{2}$ have canonical level structures of degrees 6 and 18, respectively. Write coordinates $x_{i}, i \in \mathbf{Z}_{6}$, on $\mathbf{P}^{5}$ and coordinates $y_{j}, j \in \mathbf{Z}_{18}$, on $\mathbf{P}^{17}$. Denote by $\sigma_{6}, \tau_{6}$ and $\sigma_{18}, \tau_{18}$ the generators of $\mathbf{H}_{6}$ and $\mathbf{H}_{18}$, respectively. Now $\mathbf{Z}_{6}$ acts on $E_{1} \times E_{2}$ by $(x, y) \mapsto\left(\sigma_{6}^{i}(x), \sigma_{18}^{3 i}(y)\right)$ for $i \in \mathbf{Z}_{6}$. Then $A=\left(E_{1} \times E_{2}\right) / \mathbf{Z}_{6}$ is an abelian surface which inherits a polarization coming from the product polarization on $E_{1} \times E_{2}$. Let $\pi: E_{1} \times E_{2} \rightarrow A$ be the quotient map. The linear system of $\mathbf{Z}_{6}$-invariant sections of the product polarization on $E_{1} \times E_{2}$ induces a linear system on $A$, and this in fact gives a (possibly rational) map $\alpha$ from $A$ into $\mathbf{P}^{17}(z)$ with coordinates $z_{i}, i \in \mathbf{Z}_{18}$, by mapping $E_{1} \times E_{2} \subseteq \mathbf{P}^{5}(x) \times \mathbf{P}^{17}(y)$ via

$$
z_{i}=\sum_{j \in \mathbf{Z}_{6}} x_{j} y_{3 j+i} .
$$

It is easy to see that these sections $z_{i}$ are $\mathbf{Z}_{6}$-invariant. Furthermore, the usual action of $\mathbf{H}_{18}$ on $\mathbf{P}^{17}(z)$ restricts to an action on $A$ :

$$
\begin{aligned}
\sigma_{18}(\pi(x, y)) & =\pi\left(x, \sigma_{18}(y)\right), \\
\tau_{18}(\pi(x, y)) & =\pi\left(\tau_{6}^{-1}(x), \tau_{18}(y)\right),
\end{aligned}
$$

so clearly the quotient polarization $\mathcal{L}$ on $A$ is $\mathbf{H}_{18}$-invariant and hence comes with a canonical level structure of type $(1,18)$. Now, in fact, the linear system on $A$ given by $z_{0}, \ldots, z_{17}$ is base-point-free: by [22], 10.1 .1 and 10.1 .2 , this linear system can only have base-points if there is an elliptic curve $E \subseteq A$ with $E . c_{1}(\mathcal{L})=1$. However such an elliptic curve must then pull back to a number of copies of elliptic curves on $E_{1} \times E_{2}$. But the only elliptic curves on $E_{1} \times E_{2}$, for general non-isogenous $E_{1}$, $E_{2}$, are of the form $E_{1} \times\{y\}$ or $\{x\} \times E_{2}$. While $E_{1} \times\{y\}$ is degree 6 with respect to the product polarization, its image in $A$ is also degree 6 ; so in particular, there is no elliptic curve $E \subseteq A$ with $E . c_{1}(\mathcal{L})=1$. Furthermore, by Reider's theorem ([22, 10.4.1), the morphism $\alpha: A \rightarrow \mathbf{P}^{17}$ is an embedding as long as there is no elliptic curve $E \subseteq A$ with $E . c_{1}(\mathcal{L})=2$. This is indeed the case by a similar argument, so $\alpha$ is an embedding.

Next consider the 2-torsion points of $A$. Since $\mathbf{Z}_{6} \subseteq E_{1} \times E_{2}$ contains two two-torsion points, there are two sorts of two-torsion points on $A$ : first, there are 8 points which are images of 2-torsion points on $E_{1} \times E_{2}$, and then there are 2-torsion points which are images of points on $E_{1} \times E_{2}$ satisfying

$$
-(x, y)=\left(\sigma_{6}(x), \sigma_{18}^{3}(y)\right) .
$$

(Here, the minus sign is in the group law on $E_{1} \times E_{2}$.)

In terms of projective coordinates, $x=\left(x_{0}, \ldots, x_{5}\right), y=\left(y_{0}, \ldots, y_{17}\right)$, this is saying that $\iota(x)= \pm \sigma_{6}(x)$ and $\iota(y)= \pm \sigma_{18}^{3}(y)$, as the eigenvalues of $\sigma_{6}^{-1} \circ \iota$ and $\sigma_{18}^{-3} \circ \iota$ are \pm 1 . Note there are four solutions to the first equation on $E_{1}$ and four solutions to the second on $E_{2}$, since an elliptic curve has 4 two-torsion points. If $\iota(x)=\sigma_{6}(x)$, then $\iota\left(\tau_{6}^{3}(x)\right)=-\sigma_{6}\left(\tau_{6}^{3}(x)\right)$, and if $\iota(y)=\sigma_{18}^{3}(y)$, then

$$
\iota\left(\tau_{18}^{9}(y)\right)=-\sigma_{18}^{3}\left(\tau_{18}^{9}(y)\right) .
$$


Thus solutions of these equations come in pairs, and there are two solutions each to the four equations

$$
\iota(x)=\sigma_{6}(x), \quad \iota(x)=-\sigma_{6}(x), \quad \iota(y)=\sigma_{18}^{3}(y), \quad \iota(y)=-\sigma_{18}^{3}(y) .
$$

Now if $\iota(x)=\sigma_{6}(x)$ and $\iota(y)=-\sigma_{18}^{3}(y)$, then $z:=\alpha(x, y)$ satisfies $\iota(z)=-z$. Similarly, if $\iota(x)=-\sigma_{6}(x), \iota(y)=\sigma_{18}^{3}(y)$, then $z=\alpha(x, y)$ satisfies $\iota(z)=-z$. There are a total of 8 such pairs $(x, y)$, hence 4 such points $z$, and we have identified the odd 2-torsion points of $A$. These points are in $Z_{18}$.

We now degenerate $A$ by degenerating the elliptic curve $E_{18}$ to

$$
X\left(\Gamma_{18}\right):=\bigcup_{i \in \mathbf{Z}_{18}} l_{i, i+1} \subseteq \mathbf{P}^{17},
$$

where $l_{i, i+1}$ denotes the line with equations

$$
\left\{y_{0}=y_{1}=\cdots=y_{i-1}=y_{i+2}=\cdots=y_{17}=0\right\}
$$

(see [15, §4). It is well known that such a curve is a degeneration of $\mathbf{H}_{18}$-invariant elliptic curves. Thus if we now take $A=\left(E_{1} \times X\left(\Gamma_{18}\right)\right) / \mathbf{Z}_{6}, A \cap \mathbf{P}_{-}^{7} \subseteq \overline{Z_{18}}$. We thus just need to show that if $z^{\prime} \in A \cap \mathbf{P}_{-}^{7}$, then the $4 \times 4$ Pfaffians of $M_{9}\left(\sigma \tau^{9}(z), z^{\prime}\right)$ generate the homogeneous ideal of $A$.

Let us identify such a $z^{\prime}$ : this can be taken to be the image of a point $(x, y) \in$ $E_{1} \times E_{2}$, with $x \in E_{1}$ satisfying $\iota(x)=\sigma_{6}(x)$ and $y \in E_{2}$ satisfying $\iota(y)=-\sigma_{18}^{3}(y)$. We can write $x=\left(x_{0}, x_{1}, x_{2}, x_{2}, x_{1}, x_{0}\right)$, and for $y=\left(y_{i}\right)$ we can take all coordinates $y_{i}=0$ except $y_{7}=1, y_{8}=-1$. Then the image $z$ of $(x, y)$ is

$$
z=\left(0, x_{2},-x_{2}, 0, x_{1},-x_{1}, 0, x_{0},-x_{0}, 0, x_{0},-x_{0}, 0, x_{1},-x_{1}, 0, x_{2},-x_{2}\right) .
$$

We need to identify a choice of $x_{0}, x_{1}, x_{2}$ so that $x$ is on an $\mathbf{H}_{6}$-invariant elliptic curve. To do so, recall from [15, Corollary 2.2, that the matrix $M_{3}(x, x)=$ $\left(x_{i+j} x_{i-j}+x_{i+j+3} x_{i-j+3}\right)_{0 \leq i, j \leq 2}$ has rank $\leq 1$ for any point $x \in \mathbf{P}^{5}$ contained in an $\mathbf{H}_{6}$-invariant elliptic normal curve. Restricting to the plane given by $x_{0}=$ $x_{5}, x_{1}=x_{4}, x_{2}=x_{3}$ gives the matrix

$$
\left(\begin{array}{ccc}
x_{0}^{2}+x_{2}^{2} & \left(x_{0}+x_{2}\right) x_{1} & \left(x_{0}+x_{2}\right) x_{1} \\
2 x_{1}^{2} & 2 x_{0} x_{2} & 2 x_{0} x_{2} \\
x_{0}^{2}+x_{2}^{2} & \left(x_{0}+x_{2}\right) x_{1} & \left(x_{0}+x_{2}\right) x_{1}
\end{array}\right) ;
$$

there is one independent $2 \times 2$ minor, namely $2 x_{0} x_{2}\left(x_{0}^{2}+x_{2}^{2}\right)-2 x_{1}^{3}\left(x_{0}+x_{2}\right)$.

This defines a non-singular quartic in $\mathbf{P}^{2}$, and since the moduli space of elliptic curves is one-dimensional, every point in this curve is a point on some such elliptic curve. Taking a specific point on this curve, say in characteristic $p=11,\left(x_{0}, x_{1}, x_{2}\right)=(5,6,1)$ yields a point $z^{\prime} \in \overline{Z_{18}}$. One can then calculate in Macaulay/Macaulay 2 that the Hilbert polynomial of the ideal generated by the $4 \times 4$ Pfaffians of the matrix $M_{9}\left(\sigma \tau^{9}(z), z^{\prime}\right)$ has the same Hilbert polynomials of a $(1,18)$-polarized abelian surface in $\mathbf{P}^{17}$, as desired.

While the degenerate abelian surface described by this ideal is not difficult to describe as a union of elliptic scrolls, it seems that we really do need to resort to Macaulay to verify the statement about the ideal. It seems there is no sufficiently simple choice of degeneration which does the trick for us, for if $z \in \overline{Z_{18}}$ is chosen insufficiently generally, $M_{9}\left(\sigma \tau^{9}(z), z^{\prime}\right)$ does not generate an ideal with the right Hilbert polynomial. This explains the difficulty in proving this result without appeal to computational means. 
Theorem 5.3. $\mathcal{A}_{18}^{H}$ is birational to $\tilde{\phi}(\tilde{Q})$ (see Lemma [5.1, (3), (4)), and hence in particular $\mathcal{A}_{18}^{H}$ is rational.

Proof. Lemma 5.1 shows that $(\tilde{Q}-Z) / \mathrm{GL}_{H}$ is birational to $\tilde{\phi}(\tilde{Q})$ via the map $\phi$. On the other hand, by Theorem 1.6. $\mathcal{A}_{18}^{H}$ is birational to $Z_{18} /\left(\mathrm{GL}_{H} \cap N(\mathcal{H}(1,16))\right)$. Thus to show $\mathcal{A}_{18}^{H}$ is birational to $\tilde{\phi}(\tilde{Q})$, it is enough to show that for a general point $y \in Z_{18}$,

$$
\left(\mathrm{GL}_{H} \cdot y\right) \cap Z_{18}=\left(\mathrm{GL}_{H} \cap N(\mathcal{H}(1,18))\right) \cdot y \text {. }
$$

Suppose then that there is another point $y^{\prime} \in\left(\mathrm{GL}_{H} \cdot y\right) \cap Z_{18}$ such that $y^{\prime} \notin$ $\left(\mathrm{GL}_{H} \cap N(\mathcal{H}(1,18))\right) \cdot y$. Then write $y^{\prime}=T(y)$ for some $T \in \mathrm{GL}_{H}$. Note by Proposition 1.4 that $T=\left(a_{i j}\right)$ is determined by $\alpha=a_{0,0}, \beta=a_{1,1}, \gamma=a_{0, d}$ and $\delta=a_{1, d+1}$. It is then not hard to check explicitly that

$$
M_{9}\left(\sigma \tau^{9}(x), y^{\prime}\right)=M_{9}\left(\sigma \tau^{9}(x), T(y)\right)=M_{9}\left(T^{\prime}\left(\sigma \tau^{9}(x)\right), y\right),
$$

where $T^{\prime}=\left(a_{i j}^{\prime}\right) \in \mathrm{GL}_{H}$ is determined by $a_{0,0}^{\prime}=\beta, a_{1,1}^{\prime}=\alpha, a_{0, d}^{\prime}=-\gamma, a_{1, d+1}^{\prime}=$ $-\delta$. In fact, $T^{\prime}=(\alpha \beta-\gamma \delta) T^{-1}$.

Since $y$ and $y^{\prime}$ can be assumed to be sufficiently general, it follows from Proposition 5.2 that the $4 \times 4$ Pfaffians of $M_{9}\left(\sigma \tau^{9}(x), y\right)$ and $M_{9}\left(\sigma \tau^{9}(x), y^{\prime}\right)$ generate the ideals of $(1,18)$-polarized abelian surfaces $A_{y}$ and $A_{y^{\prime}}$, respectively. Thus if we view $T^{\prime}$ as also inducing a map $T^{\prime}: \mathbf{P}^{17} \rightarrow \mathbf{P}^{17}$, we see that the $4 \times 4$ Pfaffians of $M_{9}\left(T^{\prime}\left(\sigma \tau^{9}(x)\right), y\right)$ generate the ideal of $\left(T^{\prime}\right)^{-1}\left(A_{y}\right)=T\left(A_{y}\right)$. However, this ideal coincides with that generating $A_{y^{\prime}}$, so $A_{y^{\prime}}=T\left(A_{y}\right)$ and $A_{y^{\prime}} \cong A_{y}$. This can only happen if $T \in N(\mathcal{H}(1,18)$ ) (see [15], Proposition 1.3.1), contradicting $y^{\prime} \notin\left(\mathrm{GL}_{H} \cap N(\mathcal{H}(1,18))\right) \cdot y$.

\section{Moduli of $(1,20)$-Polarized Abelian Surfaces}

We deal with the $(1,20)$ case using completely different methods, relying largely on work carried out in the second author's thesis [27] and in [3]. This approach revolves around the fact that a certain blow-up of a general $(1,20)$-polarized abelian surface in 25 points can be embedded in $\mathbf{P}^{4}$. The moduli space of these surfaces in $\mathbf{P}^{4}$ is then seen to be rational.

We begin by reviewing some material from 3 . In the discussion which follows we will have representations, both of the group $\mathbf{H}_{20}$ and the group $\mathbf{H}_{5}$. Denote the Schrödinger representations of these two groups by $V_{20}$ and $V_{5}$, respectively. We then have $\mathbf{P}^{19}=\mathbf{P}\left(V_{20} \vee\right)$, on which $\mathbf{H}_{20}$ acts, with eigenspaces of the standard involution $\iota$ yielding $\mathbf{P}_{-}^{8}, \mathbf{P}_{+}^{10} \subseteq \mathbf{P}^{19}$, and $\mathbf{P}^{4}=\mathbf{P}\left(V_{5}^{\vee}\right)$, on which $\mathbf{H}_{5}$ acts, with eigenspaces of $\iota$ yielding $\mathbf{P}_{-}^{1}, \mathbf{P}_{+}^{2} \subseteq \mathbf{P}^{4}$. We will write the standard generators $\sigma, \tau$ of $\mathbf{H}_{5}$ and $\mathbf{H}_{20}$ as $\sigma_{5}, \tau_{5}$ and $\sigma_{20}, \tau_{20}$, respectively, for clarity. We use coordinates $x_{0}, \ldots, x_{4}$ on $\mathbf{P}^{4}$ and $y_{0}, \ldots, y_{19}$ on $\mathbf{P}^{19}$, with $\sigma_{5}\left(x_{i}\right)=x_{i-1}, \tau\left(x_{i}\right)=\xi_{5}^{-i} x_{i}$, for $\xi_{5}$ a fixed primitive fifth root of unity, and similarly $\sigma_{20}\left(y_{i}\right)=y_{i-1}$ and $\tau_{20}\left(y_{i}\right)=\xi_{20}^{-i} y_{i}$ for $\xi_{20}$ a fixed 20th root of unity.

Let $\Delta \subseteq \mathbf{P}_{+}^{2}$ be the union of the six lines

$$
x_{0}=0 \text { and } x_{0}+\xi^{i} x_{1}+\xi^{-i} x_{2}=0, i \in \mathbf{Z}_{5},
$$

for $\xi$ a primitive fifth root of unity. Here we use coordinates $\left(x_{0}, x_{1}, x_{2}, x_{2}, x_{1}\right)$ on $\mathbf{P}_{+}^{2}$.

In [3], $\S 8$, given a point $a=\left(a_{0}, a_{1}, a_{2}, a_{2}, a_{1}\right) \in \mathbf{P}_{+}^{2}$, a stable rank 2 reflexive sheaf $\mathcal{E}_{a}$ is constructed. The precise details of this construction are not important; rather, only the properties are, which we summarize here. 
Definition 6.1. For a general point $a=\left(a_{0}: a_{1}: a_{2}\right) \in \mathbf{P}_{+}^{2}$, let

$$
E_{00}:=\left\{x_{1}-x_{4}=x_{2}-x_{3}=a_{0} x_{0}+2 a_{1} x_{1}+2 a_{2} x_{2}=0\right\} \subset \mathbf{P}_{+}^{2}
$$

and define $E_{i j}:=\sigma_{5}^{i} \tau_{5}^{j} E_{00}$, for $i, j \in \mathbf{Z}_{5}$ to be the translates of $E_{00}$ by the Heisenberg group $\mathbf{H}_{5}$.

Proposition 6.2. $\quad(1) \mathcal{E}_{a}$ is a stable rank 2 reflexive sheaf on $\mathbf{P}^{4}$ with Chern classes $c_{1}=-1, c_{2}=9, c_{3}=25$ and $c_{4}=50$.

(2) The singular locus $\operatorname{Sing}\left(\mathcal{E}_{a}\right)$ of the sheaf $\mathcal{E}_{a}$ (i.e., the locus where it is not locally free) consists of the 25 disjoint lines $E_{i j}, i, j \in \mathbf{Z}_{5}$. Moreover, $\mathcal{E}_{a}(3)$ is generated by sections outside the union of the lines $E_{i j}$.

(3) $h^{0}\left(\mathcal{E}_{a}(3)\right)=2$, and the zero locus of a general section $s \in H^{0}\left(\mathcal{E}_{a}(3)\right)$ is a smooth non-minimal abelian surface $\tilde{A}_{a, s} \subset \mathbf{P}^{4}$, of degree 15 , sectional genus 21, invariant under $\mathbf{H}_{5}^{e}=\mathbf{H}_{5} \rtimes\langle\iota\rangle$. The 25 lines $E_{i j}$ are exceptional lines on $\tilde{A}_{a, s}$, and they form the canonical divisor of $\tilde{A}_{a, s}$. Finally, the polarization $\mathcal{L}$ on the minimal model of $\tilde{A}_{a, s}$ is of type $(1,20)$.

Proof. See [3], $\S 8$.

Lemma 6.3. For general $a \in \mathbf{P}_{+}^{2}, s \in H^{0}\left(\mathcal{E}_{a}(3)\right)$, the action of $\mathbf{H}_{5}^{e}$ on $\mathbf{P}^{4}$ yields an action of $\mathbf{H}_{5}^{e}$ on $\tilde{A}_{a, s}$, which in turn induces an action on the minimal model $A_{a, s}$ of $\tilde{A}_{a, s}$. After a suitable choice of origin in $A_{a, s}$, the action of $\iota$ on $A_{a, s}$ is given by negation, and the action of $\mathbf{H}_{5}$ on $A_{a, s}$ is given by translation by the subgroup $\mathbf{Z}_{5} \times \mathbf{Z}_{5} \cong 4 K(\mathcal{L}) \subseteq K(\mathcal{L})$.

Proof. The action on $\tilde{A}_{a, s}$ clearly descends to an action on $A_{a, s}$, and it was already shown in the proof of 3 , Proposition 8.4, that after a suitable choice of origin, $\iota$ acts as negation. We continue to use this choice of origin. Next consider some non-trivial element $\psi \in \mathbf{H}_{5}$, so viewing $\psi$ as an automorphism of $\mathbf{P}^{4}$, it has order 5. Furthermore, it has precisely 5 fixed points in $\mathbf{P}^{4}$ which form an orbit under $\mathbf{H}_{5}$. So $\psi$ is clearly not the identity on $\tilde{A}_{a, s}$. Now $\psi$ descends to an automorphism $\psi: A_{a, s} \rightarrow A_{a, s}$ of order 5 . We first need to show that $\psi$ has no fixed points. However, clearly none of the lines $E_{i j}$ contains a fixed point of $\psi$, and by Proposition 6.2. (2), we can choose $s$ suffiently general so that $A_{a, s}$ does not contain any given finite set of points disjoint from $\bigcup E_{i j}$. Thus the action of $\psi$ on $A_{a, s}$ for $a, s$ general is fixed-point free.

Now by [7], (1.1), there is an $x \in A_{a, s}$ such that if $t_{x}$ denotes translation by $x$, then $\psi^{\prime}=t_{x} \circ \psi$ is a group automorphism of $A_{a, s}$ and the number of fixed points of $\psi^{\prime}$ and the number of fixed points of $\psi$ coincide (provided we count an infinite number of fixed points as being zero). Thus, since $\psi$ has no fixed points, we know that $\psi^{\prime}$ must have an infinite number of fixed points. There are two cases. Either $\psi^{\prime}$ is the identity or $\psi^{\prime}$ is the identity on an elliptic curve $E \subseteq A_{a, s}$. This gives an exact sequence

$$
0 \longrightarrow E \longrightarrow A_{a, s} \longrightarrow E^{\prime} \longrightarrow 0,
$$

and $\psi^{\prime}$ descends to an automorphism on the elliptic curve $E^{\prime}$. However, there is no elliptic curve with an automorphism of order 5 , so this case is ruled out. Thus $\psi^{\prime}$ is the identity and $\psi$ is a translation.

Since the only translations which preserve the line bundle $\mathcal{L}$ are translations by elements of $K(\mathcal{L})$, by definition of $K(\mathcal{L})$, we obtain the desired result. 
Theorem 6.4. (1) Let $A \subseteq \mathbf{P}^{19}$ be a general $\mathbf{H}_{20}$-invariant abelian surface embedded via a symmetric line bundle $\mathcal{L}$. Then for any odd 2-torsion point $z \in A \cap \mathbf{P}_{8}^{-}$, the subspace $V \subseteq H^{0}(A, \mathcal{L})$ of sections vanishing on an $\mathbf{H}_{5}$-orbit of $z$ (using the canonical inclusion $\mathbf{H}_{5} \subseteq \mathbf{H}_{20}$ given by $\left.\sigma_{5} \mapsto \sigma_{20}^{4}, \tau_{5} \mapsto \tau_{20}^{4}\right)$ is five-dimensional and is isomorphic to $V_{5}$ as an $\mathbf{H}_{5}$-representation. This yields a canonical identification of $\mathbf{P}^{4}=\mathbf{P}\left(V_{5}^{\vee}\right)$ and $\mathbf{P}\left(V^{\vee}\right)$. Furthermore, the rational map $A^{--\rightarrow} \mathbf{P}\left(V^{\vee}\right)$ induced by the subspace $V$ lifts to an embedding $\tilde{A} \rightarrow \mathbf{P}\left(V^{\vee}\right)$, where $\tilde{A}$ is the blow-up of $A$ along the $\mathbf{H}_{5}$-orbit of $z$. Finally, the image of $\tilde{A}$ is the zero-set of some section $s \in H^{0}\left(\mathbf{P}\left(V^{\vee}\right), \mathcal{E}_{a}(3)\right)$, for some $a \in \mathbf{P}_{+}^{2}$.

(2) If $A, A^{\prime} \subseteq \mathbf{P}^{19}$ are two such surfaces as in (1), then the images of $\tilde{A}$ and $\tilde{A}^{\prime}$ in $\mathbf{P}^{4} \cong \mathbf{P}\left(V^{\vee}\right) \cong \mathbf{P}\left(\left(V^{\prime}\right)^{\vee}\right)$ coincide if and only if the canonical level structures on $A$ and $A^{\prime}$ are $H$-equivalent, with $H=4 K(1,20)$.

Proof. Consider a non-singular surface $\tilde{A}_{a, s} \subseteq \mathbf{P}^{4}=\mathbf{P}\left(V_{5}^{\vee}\right)$ given by Proposition 6.2. Let $A_{a, s}$ be the minimal model of $\tilde{A}_{a, s}$. The surface $A_{a, s}$ carries a degree $(1,20)$ line bundle $\mathcal{L}$ with kernel $K(\mathcal{L})$. We can assume $\mathcal{L}$ is symmetric. Note that by Lemma6.3. the action of $\mathbf{H}_{5}$ on $\mathbf{P}\left(V_{5}^{\vee}\right)$ induces a symplectic identification of the group $4 K(\mathcal{L})$ with $K(1,5)$. Let $W_{20}=H^{0}\left(A_{a, s}, \mathcal{L}\right)$. After choosing a symplectic identification of $K(\mathcal{L})$ with $K(1,20)$ compatible with the identification of $4 K(\mathcal{L})$ with $K(1,5)$, we obtain a representation of $\mathbf{H}_{20}$ on $W_{20}$ so that the action of $\sigma_{5}$ on $A_{a, s}$ coming from the embedding of $\tilde{A}_{a, s}$ in $\mathbf{P}\left(V_{5}^{\vee}\right)$ coincides with $\sigma_{20}^{4}$, and similarly the action of $\tau_{5}$ coincides with $\tau_{20}^{4}$. Note that there is only one such identification up to $H$-equivalence, where $H=4 K(1,20)$, so we obtain a well-defined $H$-level structure on $A_{a, s}$.

Now $\tilde{A}_{a, s}$ is obtained by choosing a five-dimensional subspace $W_{5} \subseteq W_{20}$, and using this subspace to map $A_{a, s}$ birationally into $\mathbf{P}\left(W_{5}^{\vee}\right)$, a projective four-space. It follows however from Lemma 6.3 that in fact $W_{5}$ must be an $\mathbf{H}_{5}^{e}$-subrepresentation of $W_{20}$ coming from the natural inclusion of $\mathbf{H}_{5}^{e}$ in $\mathbf{H}_{20}^{e}$ given by $\sigma_{5} \mapsto \sigma_{20}^{4}$ and $\tau_{5} \mapsto \tau_{20}^{4}$. We also have a canonical isomorphism, up to a scalar multiple, of $V_{5}$ with $W_{5}$ as $\mathbf{H}_{5}^{e}$-representations, as these are both irreducible representations. Furthermore, the argument in [3], Proposition 8.4, says that in fact $E_{00} \subseteq \mathbf{P}_{+}^{2}$ is the image of the blow-up of an odd two-torsion point of $A_{a, s}$, and hence all the elements of $W_{5}$, as sections of $\mathcal{L}$, must vanish at one of the four odd twotorsion points of $A_{a, s}$, say $z$. Since $W_{5}$ is invariant under $\mathbf{H}_{5}$, every element of $W_{5}$ vanishes along the entire $4 K(\mathcal{L})$-orbit of $z$, which consists of 25 points. Consider the subspace $W \subseteq W_{20}$ of all sections vanishing on this orbit, so $W_{5} \subset W$. Note that $H^{0}\left(\tilde{A}_{a, s}, \mathcal{O}_{\tilde{A}_{a, s}}(1)\right) \cong W$, and so $\operatorname{dim} W>5$ contradicts linear normality of $\tilde{A}_{a, s}$. Thus $W=W_{5}$.

From this, we see that the embedding $\tilde{A}_{a, s} \subseteq \mathbf{P}\left(V_{5}^{\vee}\right)$ is uniquely determined by the choice of $H$-level structure on $A_{a, s}$ and a choice of an odd two-torsion point. However, given two different choices $z$ and $z^{\prime}$ of odd two-torsion points inducing birational maps $\phi, \phi^{\prime}: A_{a, s} \rightarrow \mathbf{P}\left(V_{5}^{\vee}\right)$, there is an $\alpha \in\left\{\sigma_{20}^{10}, \tau_{20}^{10}, \sigma_{20}^{10} \tau_{20}^{10}\right\}$ with $\alpha(z)=z^{\prime}$, and then $\phi \circ \alpha=\phi^{\prime}$. Thus the surface $\tilde{A}_{a, s}$ is determined precisely by an $H$-level structure on $A_{a, s}$. This represents only a finite number of possible choices. Since the family of surfaces $\tilde{A}_{a, s}$ parameterized by $a$ and $s$ is three-dimensional, as is the moduli space of $(1,20)$-polarized abelian surfaces, we conclude that for 
general choice of $a, s, A_{a, s}$ is general in the moduli space $\mathcal{A}_{20}$. This, together with the above description of how $\tilde{A}_{a, s}$ is obtained from $A_{a, s}$, gives (1) and (2).

Corollary 6.5. The moduli space $\mathcal{A}_{20}^{H}$ is rational.

Proof. Theorem 6.4 shows that $\mathcal{A}_{20}^{H}$ is birationally equivalent to the moduli space of pairs $(a, s)$, where $a \in \mathbb{P}_{+}^{2}$ and $s \in \mathbf{P}\left(H^{0}\left(\mathbf{P}^{4}, \mathcal{E}_{a}(3)\right)\right)$. Now the construction of the sheaf $\mathcal{E}_{a}$ given in [3] can be done in a relative fashion Zariski locally, in the sense that there is a Zariski open set $U \subseteq \mathbf{P}_{+}^{2}$ and a sheaf $\mathcal{E}$ on $U \times \mathbf{P}^{4}$ such that $\left.\mathcal{E}\right|_{\{a\} \times \mathbf{P}^{4}} \cong \mathcal{E}_{a}$ for $a \in U$. If $p_{1}, p_{2}$ are the projections of $\mathbf{P}_{+}^{2} \times \mathbf{P}^{4}$ onto the first and second factors, then it is clear that $\left.p_{1 *}\left(\mathcal{E} \otimes p_{2}^{*} \mathcal{O}_{\mathbb{P}^{4}}(3)\right)\right|_{U^{\prime}}$ is a rank two vector bundle for some open subset $U^{\prime} \subseteq U$. Thus $\mathcal{A}_{20}^{H}$ is birational to the projectivization of this rank two vector bundle. However, such a $\mathbb{P}^{1}$-bundle is a rational variety.

Proposition 6.6. (1) Let $\left\{s_{1}, s_{2}\right\}$ be a basis of $H^{0}\left(\mathcal{E}_{a}(3)\right)$. Then $V_{20, a}=\left\{s_{1} \wedge\right.$ $\left.s_{2}=0\right\} \subset \mathbf{P}^{4}$ is a quintic hypersurface containing the pencil of abelian surfaces $\left\{\tilde{A}_{a, \lambda s_{1}+\mu s_{2}} \mid(\lambda, \mu) \in \mathbf{P}^{1}\right\}$. The base locus of this pencil is the union of the lines $E_{i j}$.

(2) For a general choice of the parameter $a=\left(a_{0}: a_{1}: a_{2}\right) \in \mathbf{P}_{+}^{2}, V_{20, a}$ is the unique quintic hypersurface containing the configuration of lines $\bigcup_{i, j \in \mathbf{Z}_{5}} E_{i j}$. Furthermore, the quintic $V_{20, a}$ is $\mathbf{H}_{5}$ invariant.

Proof. (1) The fact that $V_{20, a}$ is a quintic hypersurface follows from the fact that $c_{1}\left(\mathcal{E}_{a}(3)\right)=5$, and this quintic clearly contains the given pencil of abelian surfaces. The fact that the union of lines $E_{i j}$ is the base locus of the pencil follows from Proposition 6.2, (2).

(2) We use the idea in [1, 2.2. The group $\mathbf{H}_{5}$ acts on $H^{0}\left(\mathbb{P}^{4}, \mathcal{O}_{\mathbb{P}^{4}}(5)\right)$ as a sum of characters as follows. Denote by $V_{r, s}$ the one-dimensional representation of $\mathbf{H}_{5}$ in which $\sigma$ acts by multiplication by $\xi^{r}$ and $\tau$ acts by multiplication by $\xi^{s}$, where $\xi$ is a primitive fifth root of unity. Then

$$
H^{0}\left(\mathbb{P}^{4}, \mathcal{O}_{\mathbb{P}^{4}}(5)\right)=6 V_{0,0} \oplus \bigoplus_{(r, s) \neq(0,0)} 5 V_{r, s} .
$$

Indeed, a basis of $\mathbf{H}_{5}$-invariant quintics is given by

$$
\begin{array}{rlrl}
\gamma_{0}=x_{0} x_{1} x_{2} x_{3} x_{4}, & \gamma_{1}=\sum_{i \in \mathbf{Z}_{5}} x_{i} x_{i+2}^{2} x_{i+3}^{2}, \quad \gamma_{2} & =\sum_{i \in \mathbf{Z}_{5}} x_{i}^{3} x_{i+2} x_{i+3}, \\
\gamma_{3}=\sum_{i \in \mathbf{Z}_{5}} x_{i}^{3} x_{i+1} x_{i+4}, & \gamma_{4} & =\sum_{i \in \mathbf{Z}_{5}} x_{i} x_{i+1}^{2} x_{i+4}^{2}, \quad \gamma_{5} & =\sum_{i \in \mathbf{Z}_{5}} x_{i}^{5}-5 x_{0} x_{1} x_{2} x_{3} x_{4} .
\end{array}
$$

A basis for $5 V_{r, s}$ is given by

$$
B_{r, s}=\left\{\sum_{i=0}^{4} \xi^{r i} \prod_{j=0}^{4} x_{i+j}^{m_{j}} \mid \sum_{j=0}^{4} j m_{j} \equiv-s \bmod 5, \sum_{j=0}^{4} m_{j}=5\right\} .
$$

On the other hand, by construction, $H^{0}\left(\mathcal{O}_{\cup E_{i j}}(5)\right)$ is six times the regular representation of $\mathbf{Z}_{5} \times \mathbf{Z}_{5}$, so, by Schur's Lemma, the restriction map $\rho: H^{0}\left(\mathcal{O}_{\mathbf{P}^{4}}(5)\right) \rightarrow$ $H^{0}\left(\mathcal{O}_{\cup E_{i j}}(5)\right)$ decomposes as $\rho=\bigoplus \rho_{r, s}$, where $\rho_{0,0}: 6 V_{0,0} \rightarrow 6 V_{0,0}$ and $\rho_{r, s}$ : $5 V_{r, s} \rightarrow 6 V_{r, s}$ for $(r, s) \neq(0,0)$. As a consequence, $H^{0}\left(\mathcal{I}_{\cup E_{i j}}(5)\right)=\operatorname{ker} \rho=$ $\bigoplus_{r, s} \operatorname{ker} \rho_{r, s}$. 
Thus, in order to prove (2), we check that the mappings $\rho_{r, s}$ are injective for $(r, s) \neq(0,0)$, while $\operatorname{ker} \rho_{0,0}$ is one-dimensional. For $(r, s) \neq(0,0)$, this is an open condition on $\mathbf{P}_{+}^{2}$, so it suffices to check this in a special case. We take $a_{0}=1$ and $a_{1}=a_{2}=0$. Using the above bases, this is a straightforward calculation, and we omit the details.

Now for general $a=\left(a_{0}, a_{1}, a_{2}\right) \in \mathbf{P}_{+}^{2}$, one can check that the $\mathbf{H}_{5}$-invariant quintic

$$
\begin{aligned}
\left(a_{0}^{5}-8 a_{1}^{5}-\right. & \left.8 a_{2}^{5}+15 a_{0}^{3} a_{1} a_{2}\right) \gamma_{0}+\left(a_{0}^{4} a_{1}+8 a_{1}^{3} a_{2}^{2}-4 a_{0} a_{2}^{4}\right) \gamma_{1} \\
& +\left(a_{0}^{3} a_{2}^{2}-2 a_{0}^{2} a_{1}^{3}-4 a_{0} a_{1} a_{2}^{3}\right) \gamma_{2}+\left(a_{0}^{3} a_{1}^{2}-4 a_{0} a_{1}^{3} a_{2}-2 a_{0}^{2} a_{2}^{3}\right) \gamma_{3} \\
& +\left(a_{0}^{4} a_{2}+8 a_{1}^{2} a_{2}^{3}-4 a_{0} a_{1}^{4}\right) \gamma_{4}+a_{0}^{3} a_{1} a_{2} \gamma_{5}=0
\end{aligned}
$$

contains $E_{00}$, and hence $\bigcup_{i j} E_{i j}$ by Heisenberg invariance. Thus ker $\rho_{0,0}$ is generically at least dimension one. To show it is always dimension one is then an open condition, which can again be checked at $a=(1,0,0)$. Note that in this case $V_{20, a}$ is given by the equation $x_{0} x_{1} x_{2} x_{3} x_{4}=0$.

Proposition 6.7. For a general parameter $a \in \mathbf{P}_{+}^{2}$ the quintic $V_{20, a}$ has 100 ordinary double points as singularities, four of them on each line $E_{i j}$.

Proof. We only have a computational proof of this fact. By using [5] or [10], one can easily find choices of $a$ for which $V_{20, a}$ has 100 ordinary double points as singularities. We need to show that this is actually the generic situation. By computing, again with [5] or [10], a standard basis for the Jacobian ideal, one sees that the singular locus of $V_{20, a}$ is supported on $\bigcup_{i, j \in \mathbf{Z}_{5}} E_{i j}$. Moreover, if $f$ denotes the equation of $V_{20, a}$ then

$$
\begin{array}{cr}
\frac{\partial f}{\partial x_{2}}-\frac{\partial f}{\partial x_{3}} \in I_{\mathbf{P}_{+}^{2},} & a_{0}\left(\frac{\partial f}{\partial x_{2}}+\frac{\partial f}{\partial x_{3}}\right)-2 a_{2} \frac{\partial f}{\partial x_{0}} \in I_{E_{00}}, \\
\frac{\partial f}{\partial x_{1}}-\frac{\partial f}{\partial x_{4}} \in I_{\mathbf{P}_{+}^{2},} & a_{1}\left(\frac{\partial f}{\partial x_{2}}+\frac{\partial f}{\partial x_{3}}\right)-2 a_{2} \frac{\partial f}{\partial x_{1}} \in I_{E_{00}}, \\
a_{1} \frac{\partial f}{\partial x_{0}}-a_{0} \frac{\partial f}{\partial x_{1}} \in I_{E_{00}} .
\end{array}
$$

Thus, for $a_{0} \neq 0$, the singularities of $V_{20, a}$ on $E_{00}$ are defined by $\frac{\partial f}{\partial x_{0}}$. Restricted to $E_{00}, \frac{\partial f}{\partial x_{0}}$ has simple roots, and one checks in fact that, for general choices, these points are $A_{1}$ singularities on $V_{20, a}$. Therefore, by $\mathbf{H}_{5}$ symmetry, $V_{20, a}$ has 100 nodes as singularities.

Proposition 6.8. Let $a \in \mathbf{P}_{+}^{2}$ be a general parameter and let $\widehat{V}_{20, a}$ be the small resolution of the hypersurface $V_{20, a} \subset \mathbf{P}^{4}$ obtained by blowing up one of the smooth abelian surfaces contained in the pencil traced on $V_{20, a}$. Then

(1) $\operatorname{defect}\left(V_{20, a}\right)=1$, while the topological Euler characteristic $e\left(\widehat{V}_{20, a}\right)=200-$ $2(\sharp$ nodes $)=0$.

(2) $\widehat{H}$, the pullback of a hyperplane section of $V_{20, a}$ and $\widehat{A}$, the pullback of an abelian surface in the pencil, form a basis for $\operatorname{Pic}\left(\widehat{V}_{20, a}\right) /$ tors $\cong \mathbf{Z}^{2}$.

(3) Finally, $\operatorname{dim}_{\mathbf{C}} H^{1}\left(\widehat{V}_{20, a}, \Theta_{\widehat{V}_{20, a}}\right)=h^{1}\left(\Omega_{\widehat{V}_{20, a}}^{2}\right)=2$; hence $\mathbf{P}_{+}^{2}$ is birational to the moduli of these quintic hypersurfaces. 
Proof. The calculation of the defect is easily done on [5] or [10] and is standard; we omit the details. This gives (1). Note that the calculation of the defect implies $h^{1,2}\left(\widehat{V}_{20, a}\right)=2$, from which (3) follows. The value of the topological Euler characteristic gives $h^{1,1}\left(\widehat{V}_{20, a}\right)=2$.

For (2), let $\ell$ be the class in $H^{4}\left(\widehat{V}_{20, a}, \mathbf{Z}\right)$ of the proper transform of $E_{00}$ in $\widehat{V}_{20, a}$, and let $e$ be the class of one of the exceptional curves. Then $\widehat{H} \cdot \ell=1, \widehat{H} . e=0$, and $\widehat{A} . e=-1$. Thus this intersection matrix is unimodular, so it shows that $\widehat{H}, \widehat{A}$ form a basis of $H^{2}\left(\widehat{V}_{20, a}, \mathbf{Z}\right) /$ tors $\cong \operatorname{Pic}\left(\widehat{V}_{20, a}\right) /$ tors, as desired.

Remark 6.9. (1) In light of [14], it would be interesting to compute the Brauer group of $\widehat{V}_{20, a}$. Conjecturally, it would be $\mathbf{Z} / 5 \mathbf{Z} \times \mathbf{Z} / 5 \mathbf{Z}$.

(2) The Calabi-Yau $\widehat{V}_{20, a}$ has at least three minimal models; the first is the one above obtained by blowing up one of the abelian surfaces. The 100 exceptional curves can be flopped, giving another minimal model with a pencil of non-minimal abelian surfaces, with base-locus the proper transform of $\bigcup_{i j} E_{i j}$. Finally, the curves $E_{i j}$ can be simultaneously flopped to obtain a minimal model with a basepoint-free pencil of minimal abelian surfaces. We have not, however, completely determined the Kähler cone of $\widehat{V}_{20, a}$, so there may be some additional interesting structure here.

\section{ACKNowledgments}

It is a pleasure to thank Kristian Ranestad, who joined one of us in discussions leading to some of the ideas in this paper, and Ciro Ciliberto, Wolfram Decker, Igor Dolgachev, David Eisenbud, Klaus Hulek, Nicolae Manolache, Gregory Sankaran, Frank Schreyer and Alessandro Verra from whose ideas the exposition has benefited. We are also grateful to Mike Stillman, Dave Bayer, and Dan Grayson for the programs Macaulay [5], and Macaulay2 [10, which have been extremely useful to us. Without them we would perhaps have never been bold enough to guess the existence of the structures that we describe here. Finally, we thank Philip Candelas, without whose continued interest in Calabi-Yau manifolds with small Hodge numbers this paper would probably have remained uncompleted forever.

\section{REFERENCES}

1. Aure, A., "Surfaces on quintic threefolds associated to the Horrocks-Mumford bundle", in Lecture Notes in Math., 1399 (1989), 1-9, Springer. MR1034252 (91c:14050)

2. Aure, A.B., Decker, W., Hulek, K., Popescu, S., Ranestad, K., "The Geometry of Bielliptic Surfaces in $\mathbf{P}^{4} "$, Internat. J. Math., 4 (1993), 873-902. MR,1250253 (94m:14043)

3. Aure, A.B., Decker, W., Hulek, K., Popescu, S., Ranestad, K., "Syzygies of abelian and bielliptic surfaces in $\mathbf{P}^{4} "$, Internat. J. Math. 8 (1997), no. 7, 849-919. MR1482969 (99a:14049)

4. Bak, A., Bouchard, V., Donagi, R., "Exploring a new peak in the heterotic landscape", preprint, arXiv:0811.1242.

5. Bayer, D., Stillman, M., "Macaulay: A system for computation in algebraic geometry and commutative algebra source and object code available for Unix and Macintosh computers". Contact the authors, or download from math.harvard.edu via anonymous ftp.

6. Beauville, A., "Variétés de Prym et jacobiennes intermédiares", Ann. Sci. École Norm. Sup. (4), 10 (1977), 309-391. MR0472843 (57:12532)

7. Birkenhake, Ch., Lange, H., "Fixed-point free automorphisms of abelian varieties", Geom. Dedicata, 51 (1994), 201-213. MR1293798 (95g:14052)

8. Borisov, L., Căldăraru, A., "The Pfaffian-Grassmannian derived equivalence", J. Algebraic Geom., 18 (2009), no. 2, 201-222. MR2475813(2010i:14069) 
9. Candelas, P., Davies, R., "New Calabi-Yau manifolds with small Hodge numbers", Fortschr. Phys., 58 (2010), no. 4-5, 383-466. MR 2662012

10. Grayson, D., Stillman, M., "Macaulay 2: A computer program designed to support computations in algebraic geometry and computer algebra". Source and object code available from http://www.math.uiuc.edu/Macaulay2/.

11. Griffiths, P., Harris, J., Principles of algebraic geometry, J. Wiley and Sons, New York, 1978. MR.507725 (80b:14001)

12. Gritsenko, V., "Irrationality of the moduli spaces of polarized abelian surfaces. With an appendix by the author and K. Hulek" in Abelian varieties (Egloffstein, 1993), 63-84, de Gruyter, Berlin, 1995. MR1336601 (96e:14022)

13. Gritsenko, V., "Irrationality of the moduli spaces of polarized abelian surfaces", Internat. Math. Res. Notices (1994), no. 6, 235 ff., approx. 9 pp. (electronic). MR.1277050(95c:14057)

14. Gross, M., Pavanelli, S., "A Calabi-Yau threefold with Brauer group (Z/8Z) 2 ", Proc. Amer. Math. Soc. 136 (2008), 1-9. MR2350382(2008h:14039)

15. Gross, M., Popescu, S., "Equations of $(1, d)$-polarized abelian surfaces", Math. Ann. 310 (1998) 333-377. MR1602020 (99d:14046)

16. Gross, M., Popescu, S., "The moduli space of $(1,11)$-polarized abelian surfaces is unirational", Compositio Math. 126, (2001), 1-23. MR1827859(2002c:14068)

17. Gross, M., Popescu, S., "Calabi-Yau 3-folds and moduli of abelian surfaces I", Compositio Math. 127 (2001), 169-228. MR.1845899 (2002f:14057)

18. Hori, K., Tong, D., "Aspects of non-abelian gauge dynamics in two-dimensional $\mathcal{N}=(2,2)$ theories", J. High Energy Phys. (2007). MR2318130(2009d:81351)

19. Hulek, K., Kahn, C., Weintraub, S., Moduli Spaces of Abelian Surfaces: Compactification, Degenerations, and Theta Functions, Walter de Gruyter 1993. MR 1257185 (95e:14034)

20. Hulek, K., Sankaran, G., "The Kodaira dimension of certain moduli spaces of abelian surfaces", Compositio Math., 90 (1994) 1-35. MR1266492 (95e:14035)

21. Kuznetsov, A., "Homological projective duality for Grassmannians of lines", preprint, arXiv: math/0610957.

22. Lange, H., Birkenhake, Ch., Complex abelian varieties, Springer-Verlag 1992. MR.1217487 $(94 \mathrm{j}: 14001)$

23. Melliez, F., Ranestad, K., "Degenerations of $(1,7)$-polarized abelian surfaces", Math. Scand., 97 (2005), 161-187. MR2191701 (2006i:14041)

24. Mumford, D., "On the equations defining abelian varieties", Inv. Math., 1 (1966) 287-354. MR0204427 (34:4269)

25. Mumford, D., Abelian varieties, Oxford University Press, 1974. MR0282985 (44:219)

26. Rødland, E. A., "The Pfaffian Calabi-Yau, its mirror, and their link to the Grassmannian G(2,7)", Comp. Math., 122 (2000), 135-149. MR1775415 (2001h:14051)

27. Popescu, S., On smooth surfaces of degree $\geq 11$ in the projective fourspace, Ph.D. Thesis, Saarbücken, 1993.

28. Semple, G., Roth, L., Algebraic Geometry, Chelsea 1937.

Department of Mathematics, University of California, San Diego, 9500 Gilman Drive, La Jolla, California 92093-0112

E-mail address: mgross@math.ucsd.edu

Department of Mathematics, Stony Brook University, Stony Brook, New York 117943651

Current address: Renaissance Technologies, 600 Route 25A, East Setauket, New York 11733

E-mail address: sorin@rentec.com 